\title{
QTc Interval Prolongation with Therapies Used to Treat Patients with Parkinson's Disease Psychosis: A Narrative Review
}

\author{
Yasar Torres-Yaghi \\ Amelia Carwin \\ Jacob Carolan \\ Steven Nakano \\ Fahd Amjad \\ Fernando Pagan
}

Department of Neurology, National Parkinson's Foundation Center for Excellence, Translational

Neurotherapeutics Program, Movement

Disorders Program, MedStar

Georgetown University Hospital,

Washington, DC, USA
Correspondence: Yasar Torres-Yaghi MedStar Georgetown University Hospital, Department of Neurology, National Parkinson's Foundation Center for Excellence, Translational Neurotherapeutics Program, Movement Disorders Program, 3800 Reservoir Road, NW, Washington, DC, 20007, USA

Tel +I 202-444-6087

Fax + 202-444-2186

Email yasaryaghi@gmail.com

\begin{abstract}
In addition to the classic motor symptoms of Parkinson's disease (PD), people with PD frequently experience nonmotor symptoms that can include autonomic dysfunction and neuropsychiatric symptoms such as PD psychosis (PDP). Common patient characteristics, including older age, use of multiple medications, and arrhythmias, are associated with increased risk of corrected QT interval (QTc) prolongation, and treatments for PDP (antipsychotics, dementia medications) may further increase this risk. This review evaluates how medications used to treat PDP affect QTc interval from literature indexed in the PubMed and Embase databases. Although not indicated for the treatment of psychosis, dementia therapies such as donepezil, rivastigmine, memantine, and galantamine are often used with or without antipsychotics and have minimal effects on QTc interval. Among the antipsychotics, data suggesting clinically meaningful QTc interval prolongation are limited. However, many antipsychotics have other safety concerns. Aripiprazole, olanzapine, and risperidone negatively affect motor function and are not recommended for PDP. Quetiapine is often sedating, can exacerbate underlying neurogenic orthostatic hypotension, and may prolong the QTc interval. Pimavanserin was approved by the US Food and Drug Administration (FDA) in 2016 and remains the only FDA-approved medication available to treat hallucinations and delusions associated with PDP. However, pimavanserin can increase QTc interval by approximately 5-8 $\mathrm{ms}$. The potential for QTc prolongation should be considered in patients with symptomatic cardiac arrhythmias and those receiving QT-prolonging medications. In choosing a medication to treat PDP, expected efficacy must be balanced with potential safety concerns for individual patients.
\end{abstract}

Keywords: QTc interval prolongation, antipsychotic agents, clozapine, quetiapine, pimavanserin

\section{Introduction}

Parkinson's disease (PD) is classically characterized by motor features related to central nervous system degeneration. ${ }^{1}$ Although motor symptoms are central to the diagnosis of PD, it is now recognized as a motor and nonmotor systemic illness with involvement of the autonomic nervous system and multiple major neurotransmitter systems in the brain. Nonmotor manifestations include autonomic dysfunction and neuropsychiatric symptoms (NPS), which may manifest at any stage but are particularly prevalent in advanced PD. ${ }^{2,3}$ The causes of both autonomic dysfunction and NPS are multifactorial, include disease-specific neurodegeneration, and can be exacerbated by treatments for PD. ${ }^{4-7}$ 
Approximately $70 \%-80 \%$ of people with PD experience central and peripheral autonomic dysfunction, which can affect the cardiac sympathetic and parasympathetic systems. ${ }^{8-10}$ Myocardial denervation has been observed in patients early in the course of PD disease and can lead to corrected QT interval (QTc) prolongation. ${ }^{11}$ Prolongation occurs regardless of comorbidities or coadministration of QTc-prolonging medications ${ }^{9,12}$ and appears to worsen with PD severity and age. ${ }^{13}$ QTc prolongation $>500 \mathrm{~ms}$ is particularly dangerous, as it can lead to arrhythmias, including Torsade de pointes (TdP), which is associated with sudden cardiac death. ${ }^{14}$

PD-related NPS include anxiety, depression, agitation, aggression, and psychosis. ${ }^{3,5}$ NPS are caused by alterations to a variety of neurotransmitters such as dopaminergic, serotonergic, noradrenergic, and cholinergic signaling. ${ }^{5}$ PD psychosis (PDP) affects more than $20 \%$ of all patients with $\mathrm{PD}$ and up to $70 \%$ of those with advanced $\mathrm{PD} .^{6,15}$ The hallucinations and delusions that occur with PDP are typically visual, stemming from changes in dopaminergic and serotonergic pathways, including upregulation of 5- $\mathrm{HT}_{2 \mathrm{~A}}$ signaling. ${ }^{16}$ Auditory, olfactory, and tactile hallucinations may also manifest as the disease progresses. ${ }^{6,7}$ PDP symptoms may be experienced as threatening or nonthreatening and add to patient distress and caregiver burden, such that their occurrence is a common reason for nursing home admission. ${ }^{17-19}$ Due to the lack of effective treatment options for PDP, symptom management has traditionally involved reducing the dosage of medication used to treat Parkinsonism (eg, levodopa) at the cost of the motor benefits associated with the medication. That, however, is not always practical because of exacerbation of motor symptoms. Therefore, treatment often involves managing cholinergic deficit and serotonergic dysfunction by adding a new medication as needed to alleviate remaining psychotic symptoms. ${ }^{6,15}$ Quetiapine has been the drug of choice in the past, despite a paucity of evidence supporting its use.

Although only pimavanserin is currently approved to treat hallucinations and delusions associated with $\mathrm{PDP}^{20}{ }^{20}$ a variety of antipsychotics (eg, quetiapine, clozapine, and risperidone), acetylcholinesterase inhibitors (eg, donepezil, galantamine, rivastigmine), or the N-methyl-D-aspartate (NMDA) receptor antagonist memantine are commonly used (Table 1). ${ }^{20-29}$ Data are inconsistent regarding the effects of antipsychotics on QTc interval prolongation. ${ }^{30-32}$ Some studies suggest that antipsychotics and antidementia therapies have no or minimal cardiac effect, others show clinically significant changes in QTc interval, and still others have attributed effects on QTc interval to polypharmacy or cardiac comorbidities. ${ }^{31,33,34}$

A common mechanism by which drugs prolong the QTc interval includes blocking potassium channels encoded by the human ether-A-go-go-related gene (hERG), which drives cardiac and neuronal cell repolarization. ${ }^{35,36}$ Antipsychotics, including aripiprazole, clozapine, olanzapine, pimavanserin, quetiapine, and risperidone, have been shown to be able to block or inhibit these channels in vitro. ${ }^{35,37-39}$ Acetylcholinesterase inhibitors (eg, donepezil, galantamine, rivastigmine) can affect heart function by increasing acetylcholine levels, thereby activating cardiac acetylcholine receptors, which in turn open voltage-gated calcium channels to increase intracellular calcium, which can prolong the QTc interval. ${ }^{40,41}$ Memantine blocks NMDA receptors, which when activated in the heart promote cardiac cell apoptosis and oxidative stress, and promote development of ventricular arrhythmias. ${ }^{42}$ Thus, memantine's primary effect on the heart would be expected to be cardioprotective. However, some reports have suggested that memantine treatment could promote adverse cardiovascular events in some patients, though the mechanisms at play are unclear. ${ }^{43,44}$ Given these potential mechanisms of QTc prolongation and the risk for QTc interval prolongation in older patients with PD, we reviewed the literature to better understand the relative prolongation induced by therapies commonly used in real-world settings to treat PDP.

\section{Literature Search}

PubMed and Embase databases were searched for Englishlanguage publications with available full text. No date restrictions were set. A search of titles and abstracts was conducted in December 2019 and January 2020. Both atypical antipsychotics and dementia medications regularly used to treat psychosis in people with PD were included in the search. The search string used the following terms: "QT interval prolongation" AND “aripiprazole OR clozapine OR olanzapine OR pimavanserin OR quetiapine OR risperidone OR donepezil OR galantamine OR memantine OR rivastigmine."

The authors assessed the titles and abstracts of search results for eligibility, and full text was obtained for all potentially relevant articles. Publications were included in the literature review if they described findings related to QTc interval among adults receiving at least 1 antipsychotic or dementia treatment of interest. Systematic 
Table I Medications Commonly Used to Treat Parkinson's Disease Psychosis (Name, Disease State Studied, Evidence of QT Interval Prolongation $>500 \mathrm{~ms}$, Increase in QT Interval from Baseline)

\begin{tabular}{|c|c|c|c|}
\hline Agent & $\begin{array}{l}\text { Monotherapy } \\
\text { Indication(s) in Adults }\end{array}$ & $\begin{array}{l}\text { Target Dose } \\
\text { in Adults }{ }^{\mathrm{a}}\end{array}$ & Mechanism of Action \\
\hline \multirow[t]{2}{*}{ Aripiprazole ${ }^{21}$} & Schizophrenia & $10-15 \mathrm{mg} / \mathrm{d}$ & \multirow{2}{*}{$\begin{array}{l}\text { Possibly mediated via } \mathrm{D}_{2} \text { and } 5-\mathrm{HT}_{1 \mathrm{~A}} \text { receptor partial agonism and } 5-\mathrm{HT}_{2 \mathrm{~A}} \\
\text { receptor antagonism }\end{array}$} \\
\hline & Bipolar mania & $15 \mathrm{mg} / \mathrm{d}$ & \\
\hline Clozapine $^{22}$ & Schizophrenia & $300-450 \mathrm{mg} / \mathrm{d}$ & Possibly mediated via $D_{2}$ and $5-\mathrm{HT}_{2 \mathrm{~A}}$ receptor antagonism \\
\hline \multirow[t]{2}{*}{ Olanzapine ${ }^{25}$} & Schizophrenia & $10 \mathrm{mg} / \mathrm{d}$ & \multirow[t]{2}{*}{ Possibly mediated via dopamine and $5-\mathrm{HT}_{2}$ receptor antagonism } \\
\hline & $\begin{array}{l}\text { Bipolar (mania or mixed } \\
\text { episodes) }\end{array}$ & $10-15 \mathrm{mg} / \mathrm{d}$ & \\
\hline Pimavanserin $^{20}$ & $\begin{array}{l}\text { Parkinson's disease } \\
\text { psychosis }\end{array}$ & $34 \mathrm{mg} / \mathrm{d}$ & $\begin{array}{l}\text { Possibly mediated via inverse agonist and antagonist activity at } 5-\mathrm{HT}_{2 \mathrm{~A}} \text { receptors } \\
\text { and to a lesser extent at } 5-\mathrm{HT}_{2 \mathrm{C}} \text { receptors }\end{array}$ \\
\hline \multirow[t]{3}{*}{ Quetiapine $^{29}$} & Schizophrenia & $150-750 \mathrm{mg} / \mathrm{d}$ & \multirow[t]{3}{*}{ Possibly mediated via $\mathrm{D}_{2}$ and $5-\mathrm{HT}_{2}$ receptor antagonism } \\
\hline & Bipolar mania & $400-800 \mathrm{mg} / \mathrm{d}$ & \\
\hline & Bipolar depression & $300 \mathrm{mg} / \mathrm{d}$ & \\
\hline \multirow[t]{2}{*}{ Risperidone ${ }^{24}$} & Schizophrenia & $4-8 \mathrm{mg} / \mathrm{d}$ & \multirow[t]{2}{*}{ Possibly mediated via $\mathrm{D}_{2}$ and $5-\mathrm{HT}_{2}$ receptor antagonism } \\
\hline & Bipolar mania & $\mathrm{I}-6 \mathrm{mg} / \mathrm{d}$ & \\
\hline Donepezil $^{26}$ & Alzheimer's dementia & $5-23 \mathrm{mg} / \mathrm{d}$ & \multirow{2}{*}{$\begin{array}{l}\text { Possibly increases acetylcholine concentration through reversible inhibition of its } \\
\text { hydrolysis by cholinesterase }\end{array}$} \\
\hline Galantamine $e^{27}$ & Alzheimer's dementia & $16-24 \mathrm{mg} / \mathrm{d}$ & \\
\hline Memantine $^{28}$ & Alzheimer's dementia & $\begin{array}{l}10 \mathrm{mg} \text { twice } \\
\text { daily }\end{array}$ & Possibly mediated via open-channel $\mathrm{N}$-methyl-D-aspartate receptor antagonism \\
\hline \multirow[t]{2}{*}{ Rivastigmine $^{23}$} & Alzheimer's dementia & $\begin{array}{l}3-6 \mathrm{mg} \text { twice } \\
\text { daily }\end{array}$ & \multirow[t]{2}{*}{$\begin{array}{l}\text { Possibly increases acetylcholine concentration through reversible inhibition of its } \\
\text { hydrolysis by cholinesterase }\end{array}$} \\
\hline & $\begin{array}{l}\text { Parkinson's disease } \\
\text { dementia }\end{array}$ & $\begin{array}{l}3-6 \mathrm{mg} \text { twice } \\
\text { daily }\end{array}$ & \\
\hline
\end{tabular}

Notes: a Most medications are used off label in Parkinson's disease-related psychosis (PDP). The effective dose for patients with PDP may be lower than that listed in the product label.

Abbreviation: d, day.

reviews and meta-analyses, prospective, retrospective, and observational studies, and case reports were included. Reports or studies in patients $<18$ years of age were excluded, as were reports of QTc interval prolongation following overdose and congenital long QT syndrome. Narrative review articles, preclinical studies, disease management guidelines, conference abstracts, and correspondence were also excluded. Study results and details from case reports regarding medication effects on QTc interval were extracted from all articles meeting the inclusion criteria.

Of the 463 publications returned by the database searches, 90 had available full text, met the eligibility criteria, and were included in the literature review. Due to the stringency of our search criteria, some publications that reported QTc prolongation may have been missed. In particular, the authors were aware of 2 additional relevant articles not included in the search results that presented sufficient value to be included in this review. These articles described cardiac safety (including effects on QTc interval) in patients receiving clozapine ${ }^{45}$ and donepezil. ${ }^{46}$ The literature review, therefore, included 12 systematic reviews or meta-analyses (Table 2), 54 prospective or retrospective studies (Table 3), and 26 case reports (Table 4).

The definition of QTc prolongation varied across publications. Some studies and case reports considered prolongation to occur at $\geq 500 \mathrm{~ms}$, whereas others used lower limits (eg, $>450-470 \mathrm{~ms}$ ), and still others referred to the magnitude of increase from baseline (eg, $>60 \mathrm{~ms}$ ). 
Table 2 Systematic Reviews and Meta-Analyses

\begin{tabular}{|c|c|c|c|}
\hline Reference & Patient Population & Effect on QTc Interval & Risk of QTc Prolongation \\
\hline \multicolumn{4}{|c|}{ Multiple medications } \\
\hline $\begin{array}{l}\text { Huhn et al } \\
2019^{31}\end{array}$ & Schizophrenia & $\begin{array}{l}\text { Mean difference }(95 \% \mathrm{Cl}) \text { vs placebo: } \\
\text { Aripiprazole: }-0.43 \mathrm{~ms}(-3.62 \text { to } 2.77) \\
\text { Olanzapine: } 4.29 \mathrm{~ms}(1.9 \mathrm{l}-6.68) \\
\text { Quetiapine: } 3.43 \mathrm{~ms}(0.94-6.00) \\
\text { Risperidone: } 4.77 \mathrm{~ms}(2.68-6.87)\end{array}$ & NR \\
\hline $\begin{array}{l}\text { Aronow } \\
\text { et al } 2018^{30}\end{array}$ & Mental disorders & $\begin{array}{l}\text { SMD }(95 \% \mathrm{Cl}) \text { vs placebo: } \\
\text { Aripiprazole: NR } \\
\text { Olanzapine: }-0.14 \text { (-0.29 to } 0.01) \\
\text { Quetiapine: } 0.67(0.14-1.19) \\
\text { Risperidone: } N R\end{array}$ & $\begin{array}{l}\text { RR }(95 \% \mathrm{Cl}) \text { vs placebo: } \\
\text { Aripiprazole: } 0.89(0.08-9.8 \mathrm{I}) \\
\text { Olanzapine: } 0.34(0.16-0.70) \text { to } 0.46(0.04-4.96) \\
\text { Quetiapine: } 2.87(0.12-70.08) \\
\text { Risperidone: } 0.62(0.09-4.4 \mathrm{I}) \text { to } 1.90(1.29-2.79)\end{array}$ \\
\hline $\begin{array}{l}\text { Takeuchi } \\
\text { et al } 2015^{59}\end{array}$ & $\begin{array}{l}\text { Schizophrenia or } \\
\text { schizoaffective } \\
\text { disorder concurrently } \\
\text { using } \geq 2 \text { APs }\end{array}$ & $\begin{array}{l}\text { Mean change from baseline: } \\
\text { Clozapine + risperidone: }-1 \mathrm{l} \text { to }-10 \mathrm{~ms} \\
\text { Clozapine + aripiprazole: } 4 \mathrm{~ms} \\
\text { Clozapine + placebo: }-3 \text { to } 13 \mathrm{~ms} \\
\text { Risperidone alone: }-8 \mathrm{~ms}\end{array}$ & NR \\
\hline $\begin{array}{l}\text { Asmal et al } \\
2013^{87}\end{array}$ & Schizophrenia & $\begin{array}{l}\text { Mean difference }(95 \% \mathrm{Cl}) \text { : } \\
\text { Quetiapine vs olanzapine: } 4.8 \mathrm{I}(0.34-9.28) \\
\text { Quetiapine vs risperidone: } 2.21 \text { (-5.05 to } 9.48)\end{array}$ & $\begin{array}{l}\text { RR }(95 \% \mathrm{Cl}) \text { vs active comparator: } \\
\text { Quetiapine vs aripiprazole: } 3.21(0.13-76.74) \\
\text { Quetiapine vs olanzapine: } 12.96(0.73-229.17) \\
\text { Quetiapine vs risperidone: } 1.34(0.36-5.04)\end{array}$ \\
\hline $\begin{array}{l}\text { Leucht et al } \\
2013^{57}\end{array}$ & $\begin{array}{l}\text { Schizophrenia or } \\
\text { related disorders }\end{array}$ & $\begin{array}{l}\text { SMD }(95 \% \mathrm{Cl}) \text { : } \\
\text { Aripiprazole vs placebo: } 0.0 \mathrm{I}(-0.13 \text { to } 0.15) \\
\text { Aripiprazole vs quetiapine: }-0.17(-0.33 \text { to } 0.0 \mathrm{I}) \\
\text { Aripiprazole vs risperidone: }-0.25(-0.40 \text { to }-0.10) \\
\text { Aripiprazole vs olanzapine: }-0.2 \mathrm{I}(-0.37 \text { to }-0.05) \\
\text { Olanzapine vs placebo: }-0.22(-0.3 \mathrm{I} \text { to }-0.1 \mathrm{I}) \\
\text { Olanzapine vs risperidone: }-0.04(-0.16 \text { to } 0.09) \\
\text { Quetiapine vs placebo: } 0.17(0.06-0.29) \\
\text { Quetiapine vs olanzapine: }-0.04(-0.18 \text { to } 0.1 \mathrm{I}) \\
\text { Quetiapine vs risperidone: }-0.08(-0.22 \text { to } 0.06) \\
\text { Risperidone vs placebo: }-0.25(-0.36 \text { to }-0.15)\end{array}$ & $\begin{array}{l}\text { OR }(95 \% \mathrm{Cl}) \text { : } \\
\text { Aripiprazole: } 0.0 \mathrm{I}(-0.13 \text { to } 0.15) \\
\text { Olanzapine: } 0.22(0.1 \mathrm{I}-0.3 \mathrm{I}) \\
\text { Quetiapine: } 0.17(0.06-0.29) \\
\text { Risperidone: } 0.25(0.15-0.36)\end{array}$ \\
\hline $\begin{array}{l}\text { Chung et al } \\
2011^{56}\end{array}$ & Schizophrenia & $\begin{array}{l}\text { Mean difference }(95 \% \mathrm{Cl}) \text { vs placebo or other APs: } \\
\text { Aripiprazole: }-2.49(-6.87 \text { to I.88; } P=0.26) \\
\text { Olanzapine: } 0.38(-3.05 \text { to } 3.8 \mathrm{I}) \\
\text { Risperidone: } 2.5 \mathrm{I}(-0.10 \text { to } 5 . \mathrm{I} \mathrm{I})\end{array}$ & $\begin{array}{l}\text { RR }(95 \% \mathrm{Cl}): \\
\text { Aripiprazole: } 0.33(0.12-0.93) \\
\text { Olanzapine: } 0.84 \text { (0.44-I.58) } \\
\text { Risperidone: not available }\end{array}$ \\
\hline \multicolumn{4}{|l|}{ Aripiprazole } \\
\hline $\begin{array}{l}\text { Polcwiartek } \\
\text { et al } 2015^{58}\end{array}$ & $\begin{array}{l}\text { Patients with risk } \\
\text { factors for TdP }\end{array}$ & $\begin{array}{l}\text { Mean difference }(95 \% \mathrm{Cl}) \text { vs placebo: } \\
-0.73(-0.87 \text { to }-0.6 ; P<0.00 \mathrm{I})\end{array}$ & $\begin{array}{l}\text { RR }(95 \% \mathrm{Cl}) \text { vs placebo: } \\
0.85(0.5 \mathrm{I}-\mathrm{I} .43 ; P=0.54) \\
\text { RR }(95 \% \mathrm{Cl}) \text { vs other antipsychotics: } \\
0.53(0.32-0.88 ; P=0.0 \mathrm{I})\end{array}$ \\
\hline \multicolumn{4}{|l|}{ Olanzapine } \\
\hline $\begin{array}{l}\text { Kishi et al } \\
2015^{88}\end{array}$ & Agitation & $\begin{array}{l}\text { SMD }(95 \% \mathrm{Cl}) \text { vs placebo: } \\
-0.14(-0.29 \text { to } 0.01 ; P=0.08)\end{array}$ & $\begin{array}{l}\text { RR }(95 \% \mathrm{Cl}) \text { vs placebo: } \\
0.34(0.16-0.70 ; P=0.003)\end{array}$ \\
\hline
\end{tabular}

(Continued) 
Table 2 (Continued).

\begin{tabular}{|c|c|c|c|}
\hline Reference & Patient Population & Effect on QTc Interval & Risk of QTc Prolongation \\
\hline \multicolumn{4}{|l|}{ Risperidone } \\
\hline $\begin{array}{l}\text { Rabkin, } \\
2014^{122}\end{array}$ & $\begin{array}{l}\text { Patients across } \\
\text { multiple age decades }\end{array}$ & $\begin{array}{l}\text { Little effect on QTc, but a trend of increasing QTc } \\
\text { with age: person in mid-70s beginning risperidone } \\
\text { with QTc of I SD greater than mean would have } \\
\text { treatment response I SD greater than average } \\
\text { response to risperidone }\end{array}$ & NR \\
\hline $\begin{array}{l}\text { Cartwright } \\
\text { et al } 2013^{120}\end{array}$ & $\begin{array}{l}\text { People receiving } \\
\text { risperidone }\end{array}$ & 0.6 to $13.9 \mathrm{~ms}$ increase from baseline & $\begin{array}{l}0-50 \% \text { of patients with QTc }>420 \text { ms or changes } \\
\geq 30 \text { ms; higher proportion in patients with poor } \\
\text { risperidone metabolism, ie, higher plasma } \\
\text { concentration }\end{array}$ \\
\hline $\begin{array}{l}\text { Gopal et al } \\
2013^{121}\end{array}$ & $\begin{array}{l}\text { Patients in registration } \\
\text { studies of risperidone }\end{array}$ & NR & $\begin{array}{l}\text { QTcF }>60 \mathrm{~ms} \text { and } \geq 500 \mathrm{~ms} \text { in } 0.1 \% \text { of patients } \\
\text { receiving risperidone and } 0.1 \% \text { receiving placebo; } \\
\text { all of these patients }>74 \text { years old }\end{array}$ \\
\hline $\begin{array}{l}\text { Rattehalli } \\
\text { et al } 2016^{123}\end{array}$ & Schizophrenia & NR & $\begin{array}{l}\text { RR }(95 \% \mathrm{Cl}): \\
8.46(1.07-67.07)\end{array}$ \\
\hline
\end{tabular}

Abbreviations: AP, antipsychotic; Cl, confidence interval; OR, odds ratio; NR, not reported; QTc, corrected QT interval; QTcF, Fridericia-corrected QT interval; RR, risk ratio; SD, standard deviation; SMD, standardized mean difference; TdP, Torsades de pointes.

\section{Antipsychotics}

Most publications reported on multiple antipsychotics rather than assessing the effects of a single drug and evaluated treatments in people with schizophrenia, not PDP. The magnitude of effect on QTc interval and risk of prolongation $>500 \mathrm{~ms}$ varied across antipsychotics and between studies. Analyses reported a 0.4-ms reduction in QTc interval with aripiprazole and a 3- to 6-ms increase with quetiapine, olanzapine, and risperidone, ${ }^{31}$ with quetiapine and olanzapine associated with increases greater than those with risperidone $(P<0.01) .{ }^{47}$ In contrast, 1 study found no QTc prolongation with olanzapine, quetiapine, or risperidone, ${ }^{48}$ with similar rates of change in QTc interval among these 3 medications ( -0.0099 to 0.0030 $\mathrm{ms} /$ day). ${ }^{49}$

Many factors can affect a patient's QTc intervals, including cardiac and hepatic comorbidities, use of QTcprolonging medications, older age, female sex, and time of day. ${ }^{50-52}$ The choice of antipsychotic may contribute to $17 \%-55 \%$ of a patient's response, with $10 \%-12 \%$ of this variation attributed to genetic expression. ${ }^{53}$

In general, QTc prolongation appears to occur infrequently with atypical antipsychotics. A search of the World Health Organization pharmacovigilance database found that, as of January 2010, 489 reports exist on QT prolongation, TdP, and/or cardiac arrest related to olanzapine, and 520 reports exist on QT prolongation, TdP, and/ or cardiac arrest related to quetiapine since the medication approvals in 1996 and 1995, respectively. ${ }^{54}$ Analysis of a database encompassing Austria, Germany, and Switzerland reported a QTc prolongation frequency of $0.006 \%$ among patients treated with clozapine or quetiapine and $0 \%$ with aripiprazole, olanzapine, and risperidone. $^{55}$

\section{Aripiprazole}

Aripiprazole was mentioned in 6 systematic reviews and meta-analyses, ${ }^{30,31,56-59} 13$ prospective or observational studies, ${ }^{33,47,55,60-69}$ and 4 case reports. ${ }^{70-73}$ The literature consistently showed that aripiprazole is associated with small reductions in QTc interval. Four meta-analyses of aripiprazole described reduced mean QTc intervals $(-0.43$ to $-3.38 \mathrm{~ms}$ ) and reduced risk of prolongation with aripiprazole when compared with placebo (risk ratios [RR], 0.33 [95\% confidence interval (CI), $0.12-0.93$ ] to 7.58 [0.40-143.03]; Table 2). ${ }^{30,31,56,58}$

Six studies of 1351 patients with psychiatric disorders reported no clinically significant QTc interval prolongation with aripiprazole (Table 3). ${ }^{60,62,63,65,66,74}$ These findings were supported by a cross-sectional study that controlled for patient age, sex, diagnosis, length of illness, setting (inpatient vs outpatient), use of mood stabilizers or antidepressants, number of antipsychotics, and antipsychotic dose. ${ }^{33}$ In that study, aripiprazole was associated with a 


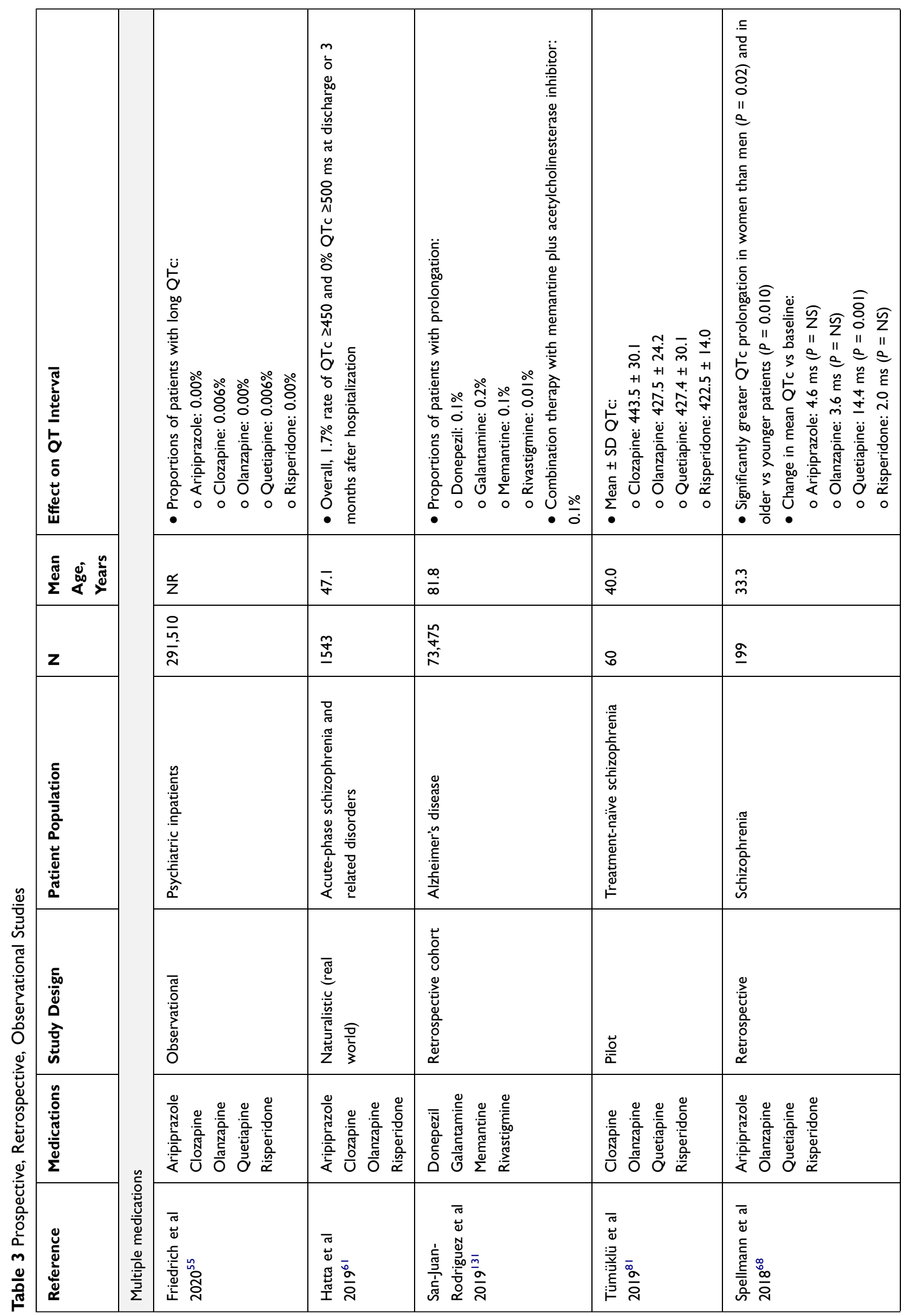




\begin{tabular}{|c|c|c|c|c|}
\hline 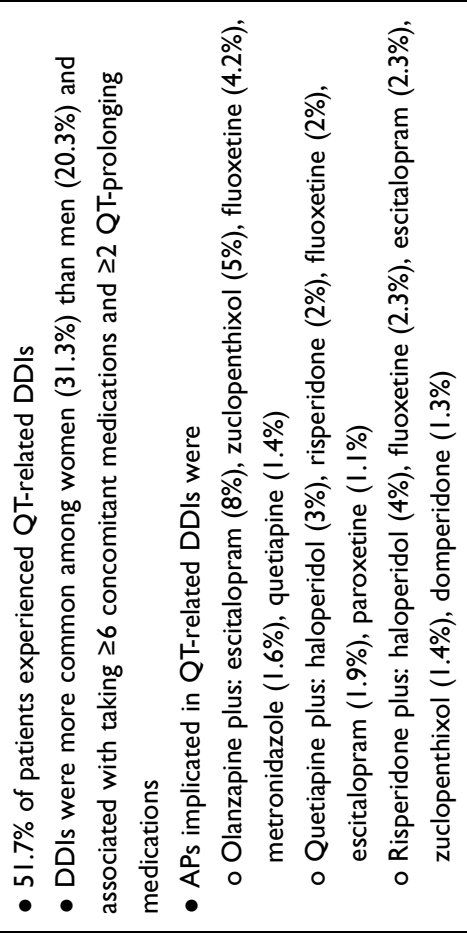 & 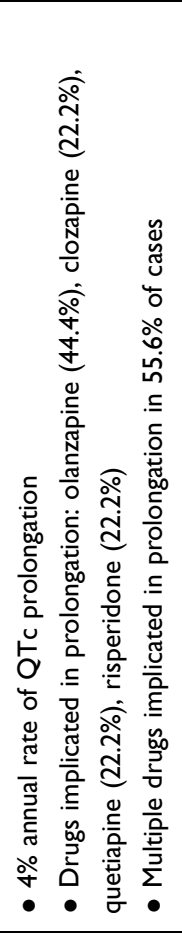 & 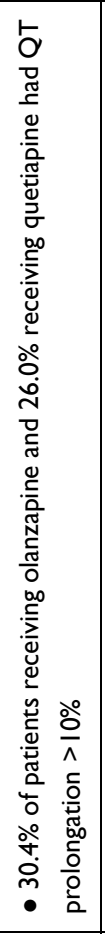 & 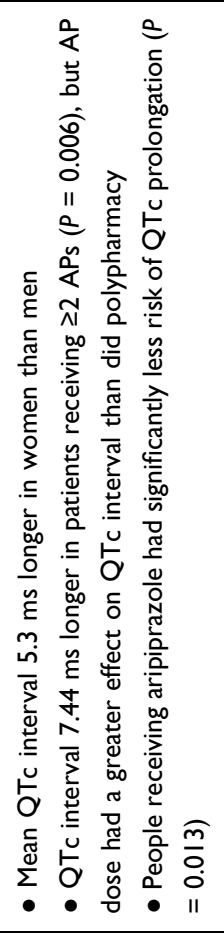 & 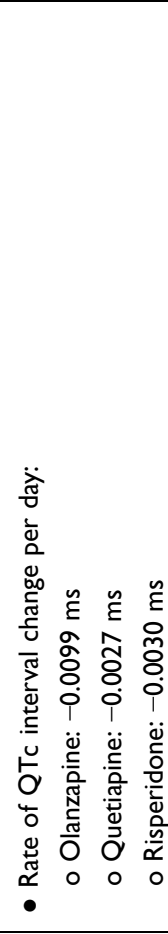 \\
\hline ณึ & 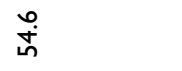 & $\stackrel{\alpha}{Z}$ & 兑 & $\overline{\dot{m}}$ \\
\hline 8 & $\stackrel{\stackrel{\sim}{*}}{ }$ & : : & $\stackrel{\breve{N}}{N}$ & 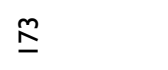 \\
\hline 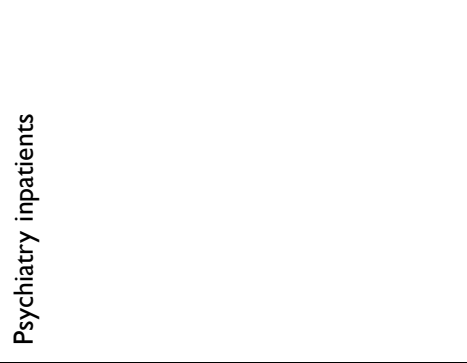 & 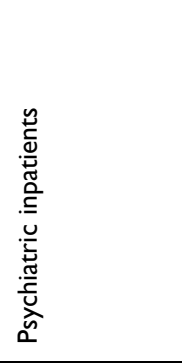 & 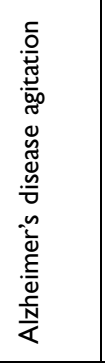 & 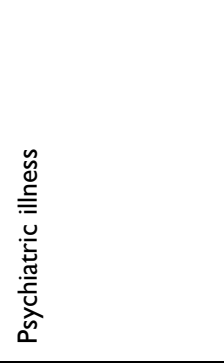 & 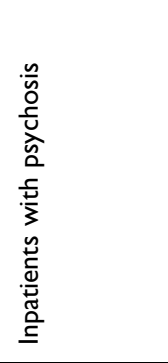 \\
\hline 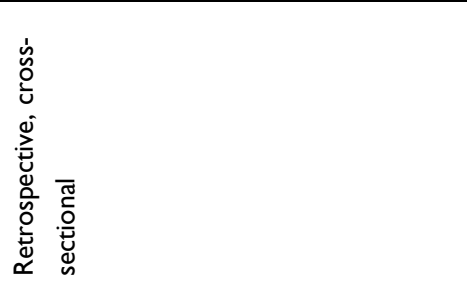 & 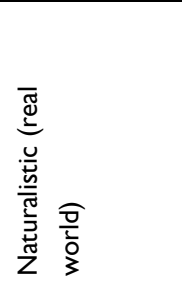 & 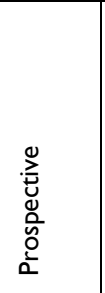 & 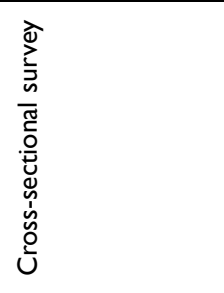 & 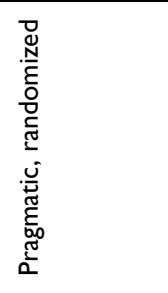 \\
\hline 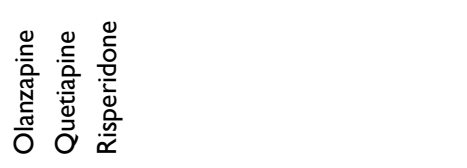 & 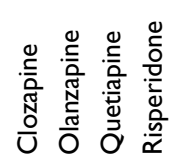 & 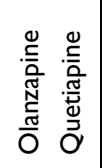 & 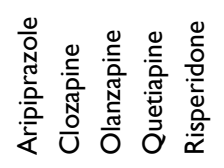 & 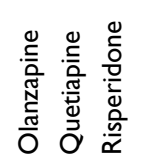 \\
\hline 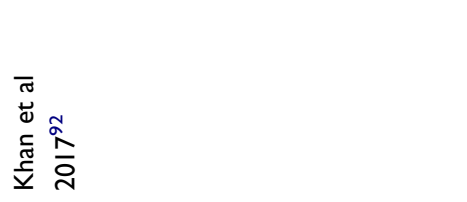 & 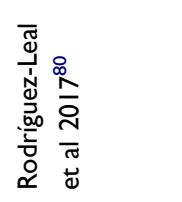 & 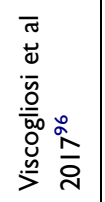 & 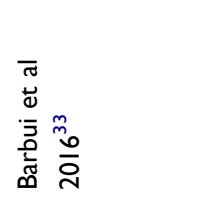 & 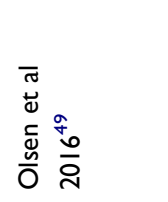 \\
\hline
\end{tabular}




\begin{tabular}{|c|c|c|c|c|c|}
\hline 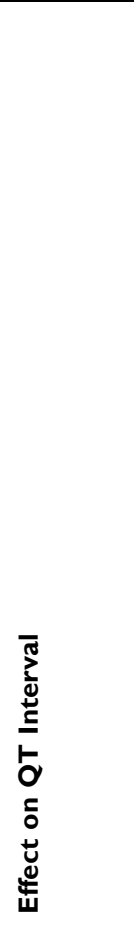 & 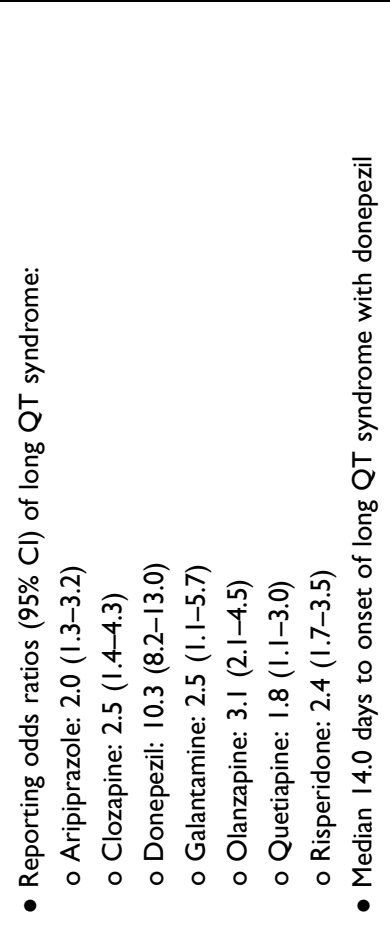 & 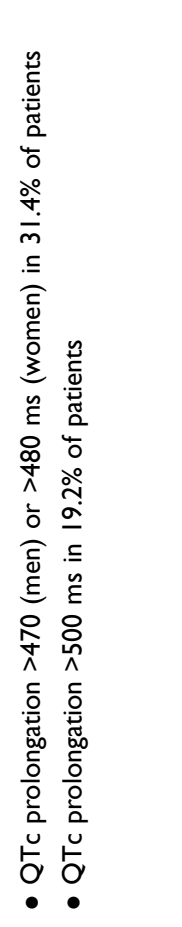 & 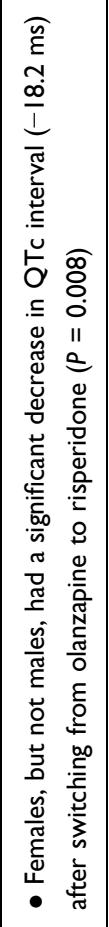 & 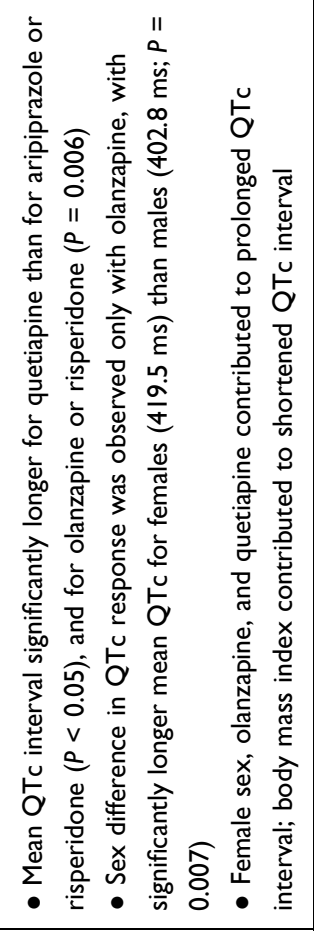 & 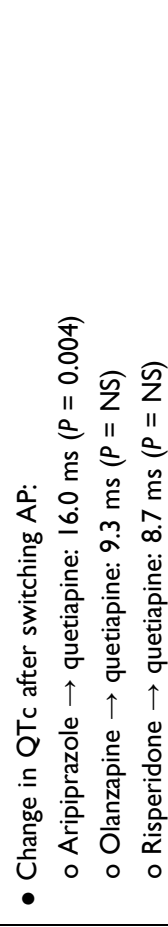 \\
\hline 尔 & & $\frac{n !}{6}$ & $\begin{array}{l}\text { aे } \\
\text { iे }\end{array}$ & 岀 & స్ \\
\hline z & & 으 & $\bar{\sim}$ & สี & 구 \\
\hline 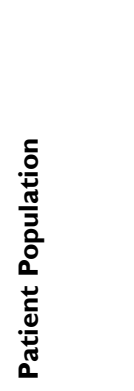 & 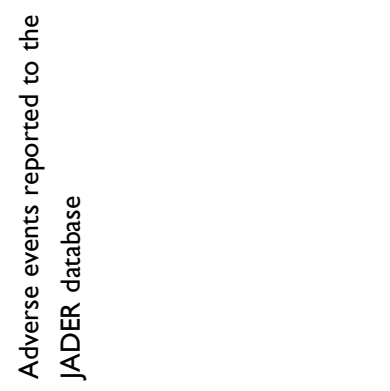 & 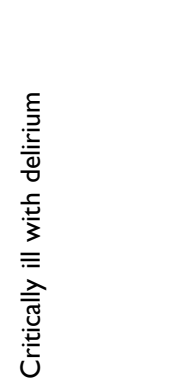 & 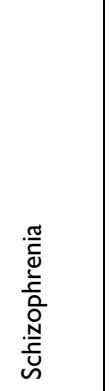 & 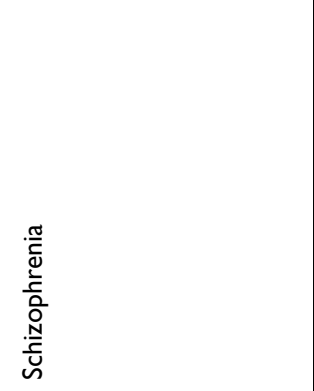 & 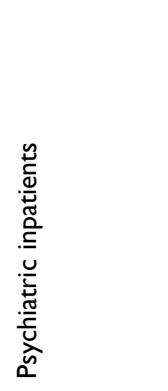 \\
\hline 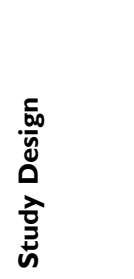 & 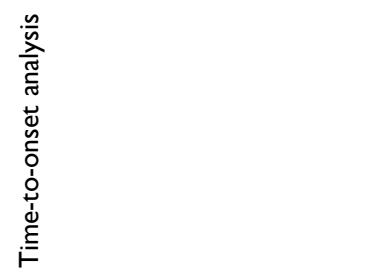 & 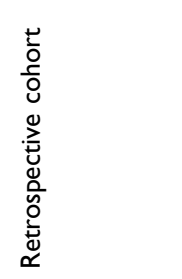 & 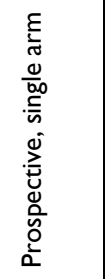 & 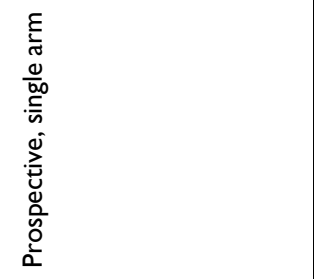 & 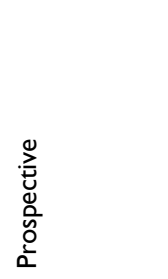 \\
\hline 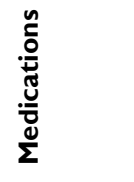 & 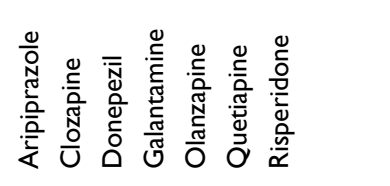 & 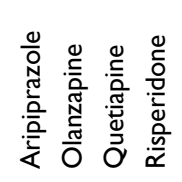 & 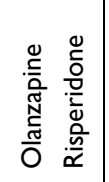 & 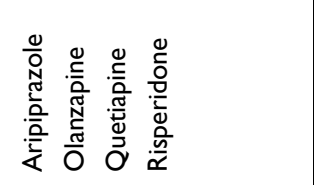 & 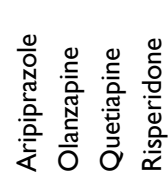 \\
\hline 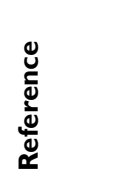 & 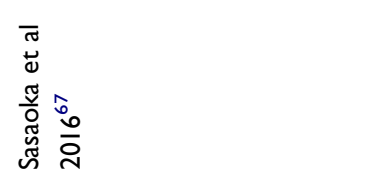 & 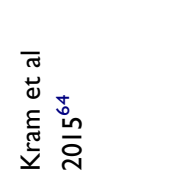 & 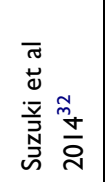 & 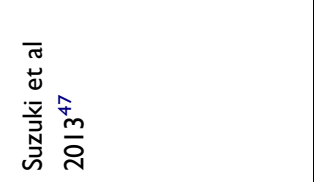 & 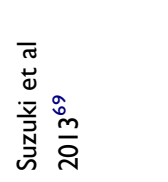 \\
\hline
\end{tabular}




\begin{tabular}{|c|c|c|c|c|c|}
\hline 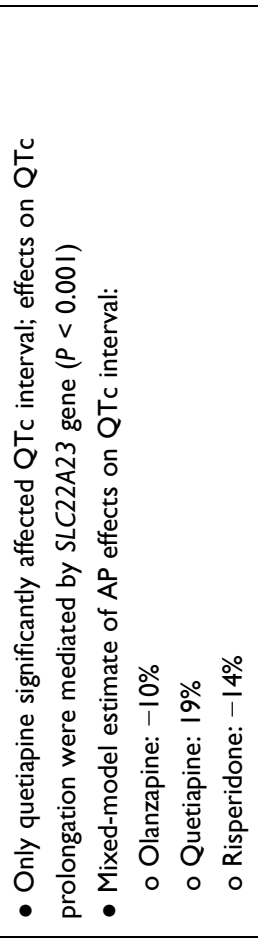 & 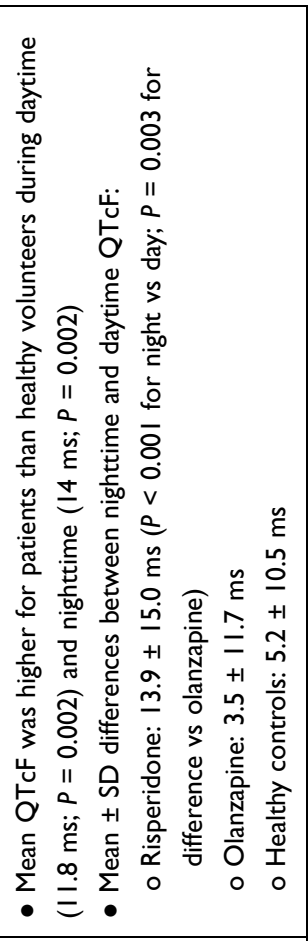 & 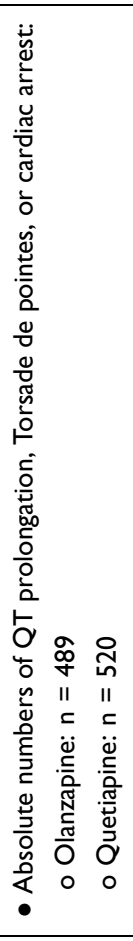 & 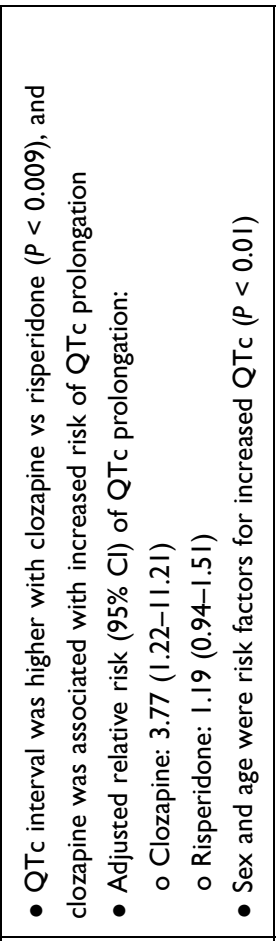 & 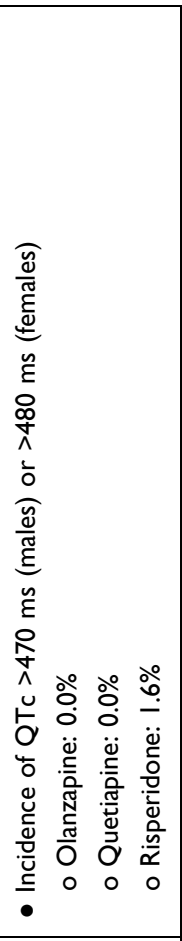 & 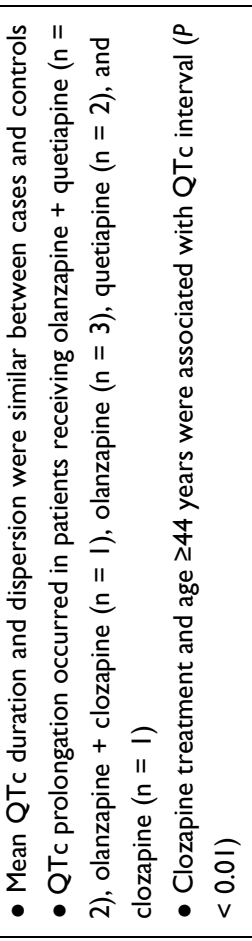 \\
\hline 苂 & $\begin{array}{l}\stackrel{\circ}{\circ} \\
\text { ch }\end{array}$ & $\begin{array}{l}\hat{y} \\
\dot{y}\end{array}$ & กี & $\frac{\alpha}{Z}$ & $\stackrel{m}{\stackrel{m}{\sigma}}$ \\
\hline$\stackrel{\infty}{\Re}$ & 으 & $\begin{array}{l}\stackrel{\circ}{\circ} \\
\text { oे }\end{array}$ & 守 & $\stackrel{\stackrel{0}{*}}{\sigma}$ & $\Xi$ \\
\hline 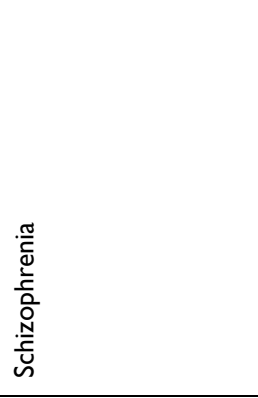 & 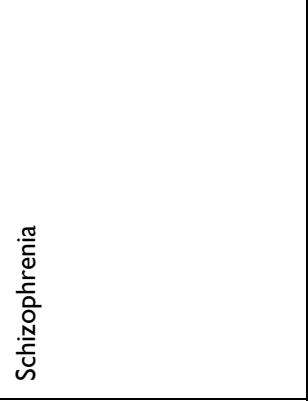 & 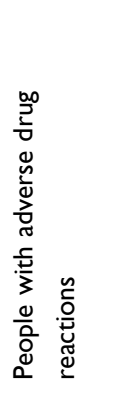 & 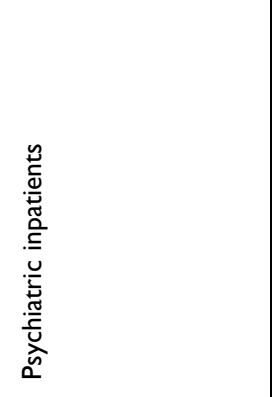 & 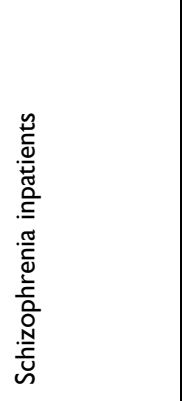 & 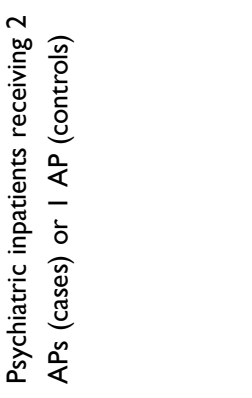 \\
\hline 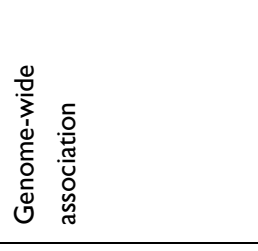 & 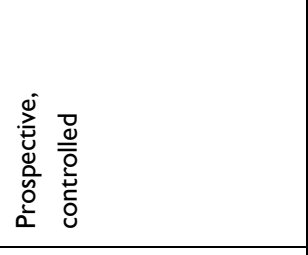 & 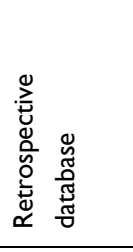 & 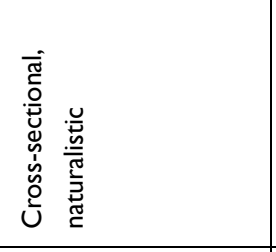 & 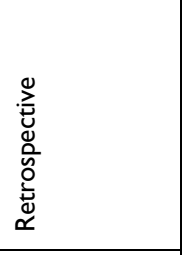 & 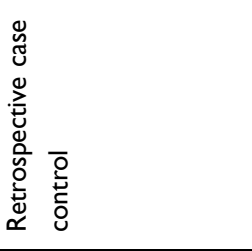 \\
\hline 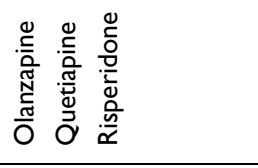 & 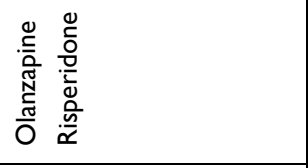 & 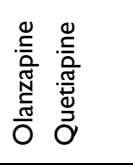 & 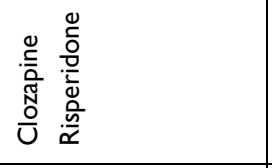 & 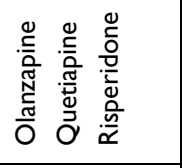 & 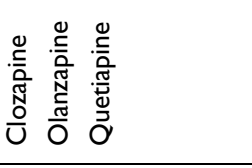 \\
\hline 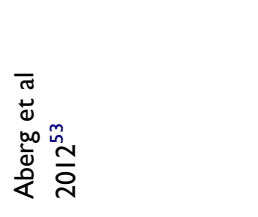 & 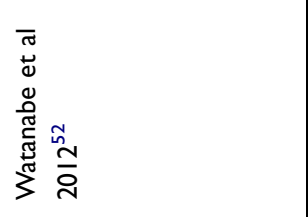 & 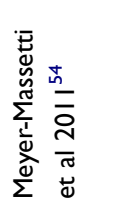 & 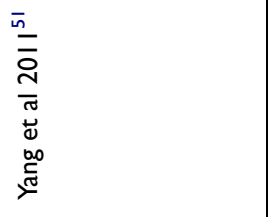 & 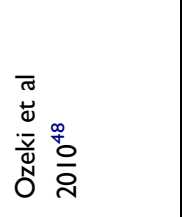 & 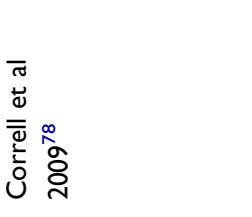 \\
\hline
\end{tabular}




\begin{tabular}{|c|c|c|c|c|c|c|c|c|c|}
\hline 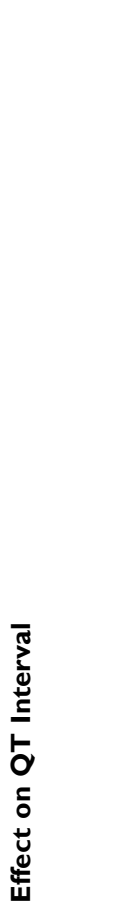 & 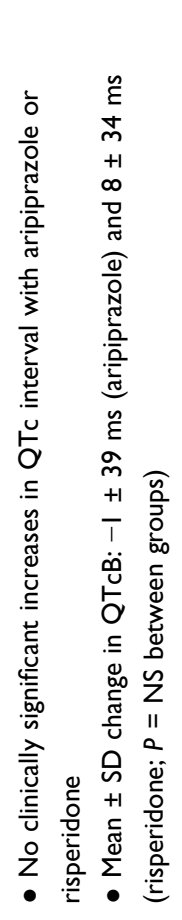 & 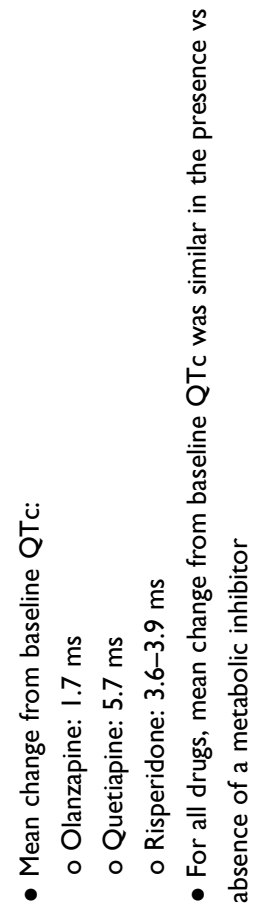 & 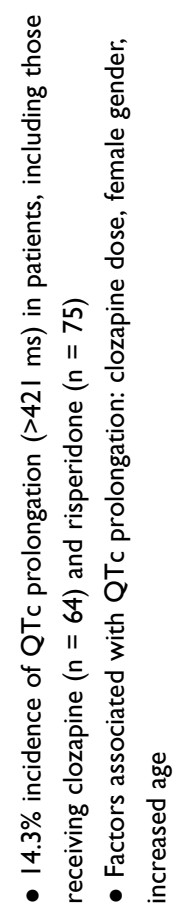 & 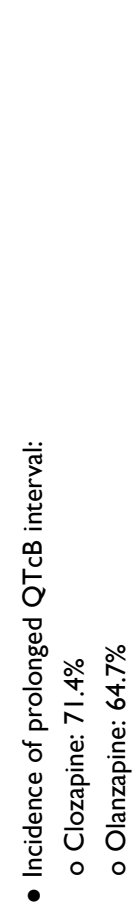 & & 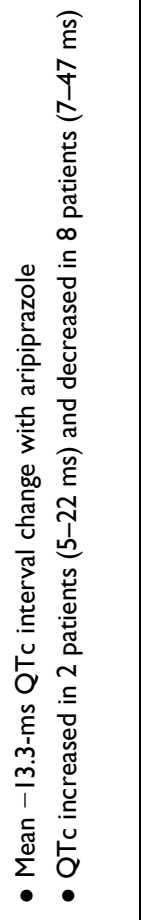 & 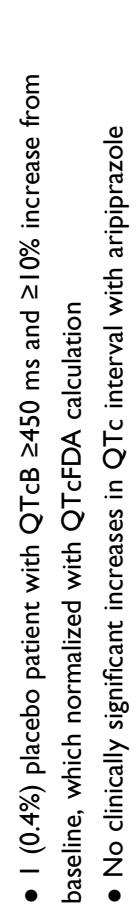 & 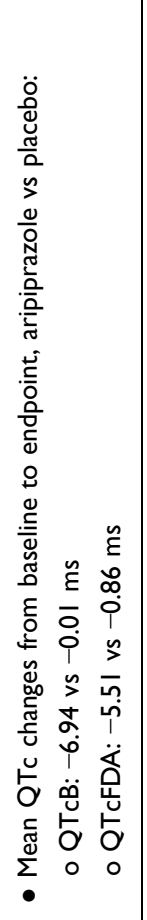 & 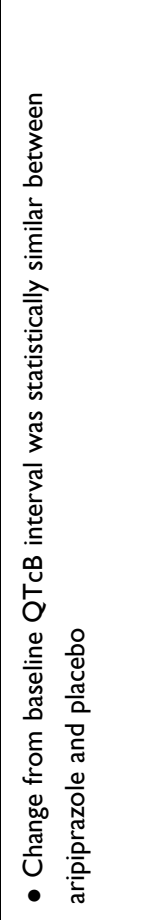 \\
\hline 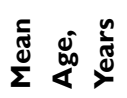 & $\stackrel{\stackrel{n}{m}}{n}$ & $\begin{array}{l}\stackrel{2}{\rho} \\
\stackrel{p}{\infty} \\
\text { pon }\end{array}$ & $\underset{m}{m}$ & 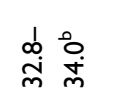 & & $\stackrel{m}{R}$ & $\stackrel{n}{\dot{q}}$ & $\underset{\text { ㄱ }}{\text { G }}$ & $\stackrel{\circ}{\infty}$ \\
\hline$z$ & œ & $\stackrel{\circ}{\circ}$ & $\stackrel{N}{\sigma}$ & $\stackrel{\circ}{\infty}$ & & 으 & స్ & $\frac{\circ}{m}$ & $\frac{\sigma}{\sigma}$ \\
\hline 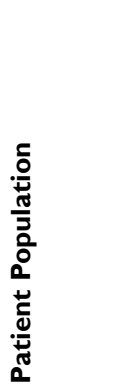 & 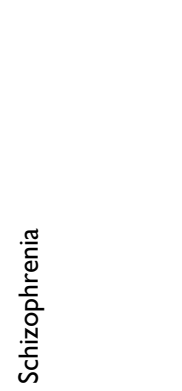 & 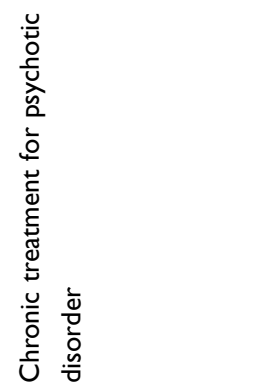 & 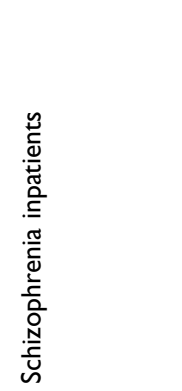 & 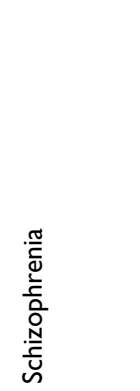 & & 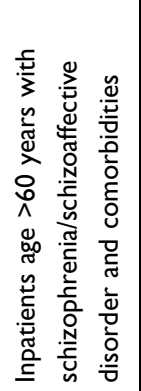 & 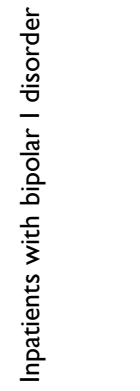 & 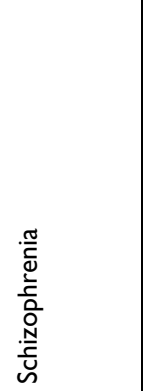 & 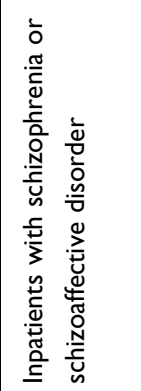 \\
\hline 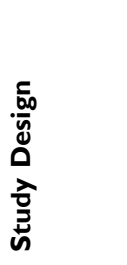 & 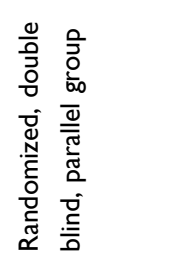 & 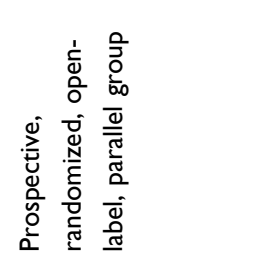 & 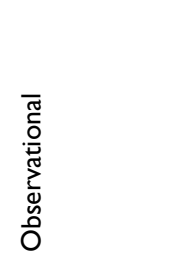 & 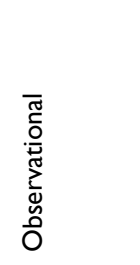 & & 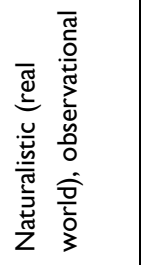 & 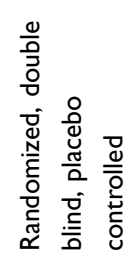 & 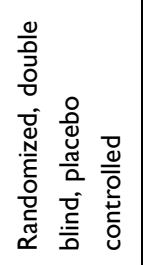 & 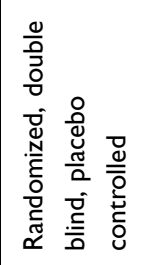 \\
\hline 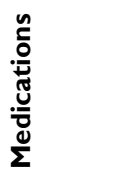 & 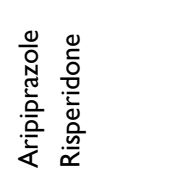 & 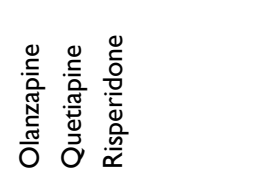 & 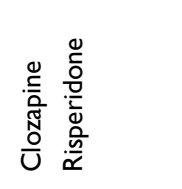 & 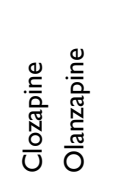 & & $\begin{array}{l}\frac{0}{0} \\
\frac{0}{\frac{\pi}{2}} \\
\frac{20}{\frac{2}{2}}\end{array}$ & 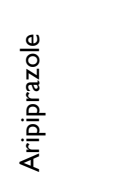 & 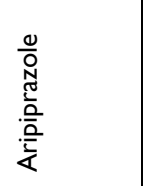 & $\begin{array}{l}\frac{0}{2} \\
\frac{N}{0} \\
\frac{.0}{0.0} \\
\frac{0}{<}\end{array}$ \\
\hline 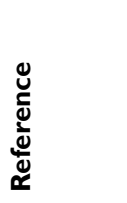 & 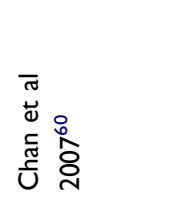 & 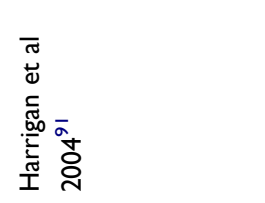 & 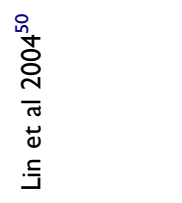 & 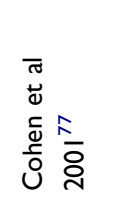 & $\begin{array}{l}\frac{0}{0} \\
\frac{N}{0} \\
\frac{0}{2} \\
\frac{2}{2} \\
\frac{2}{<}\end{array}$ & 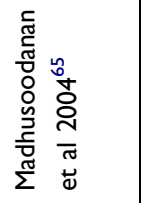 & 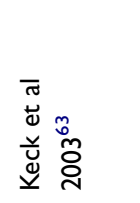 & 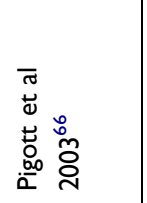 & 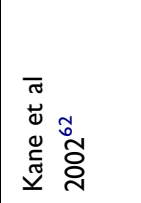 \\
\hline
\end{tabular}




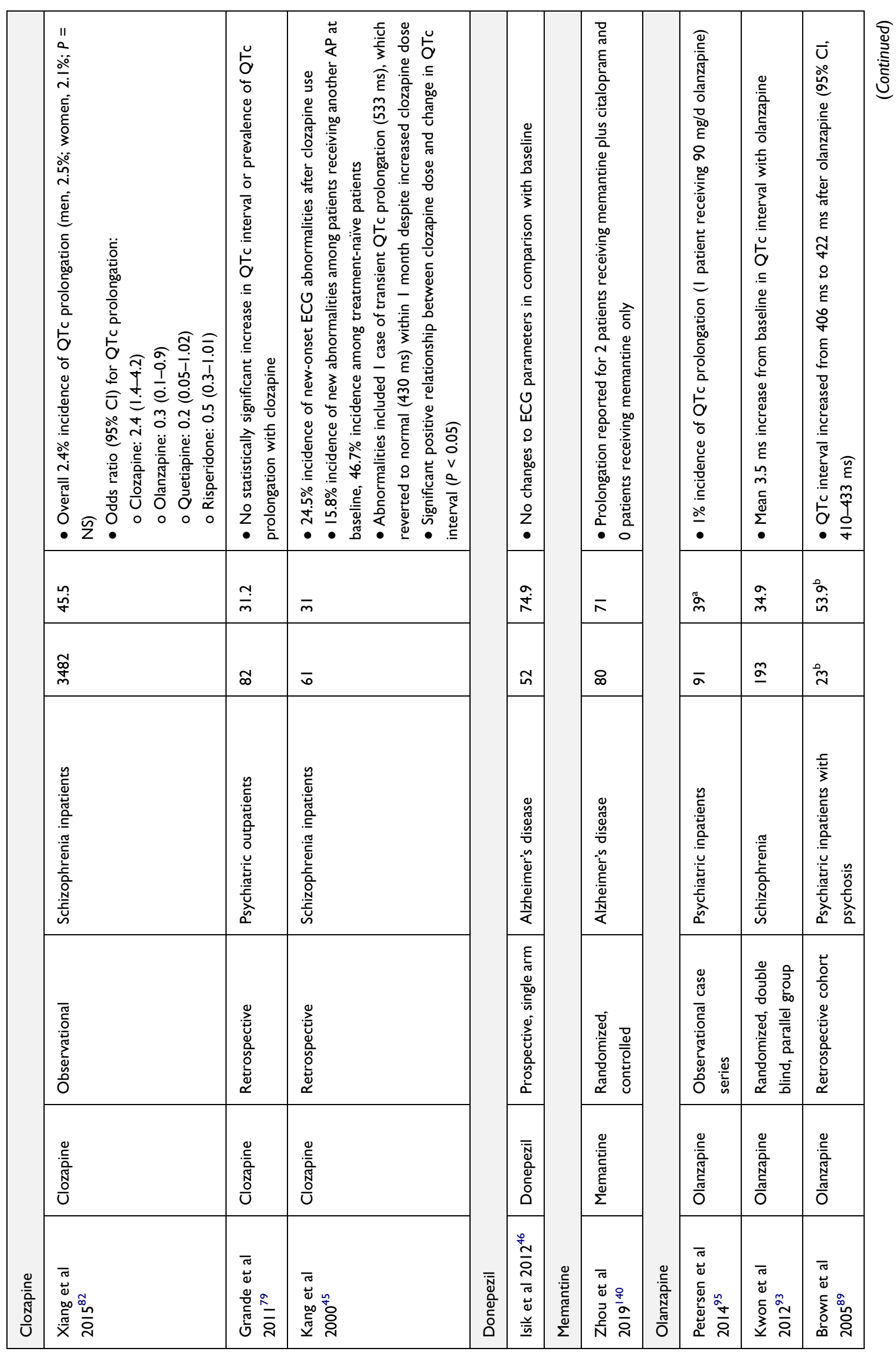




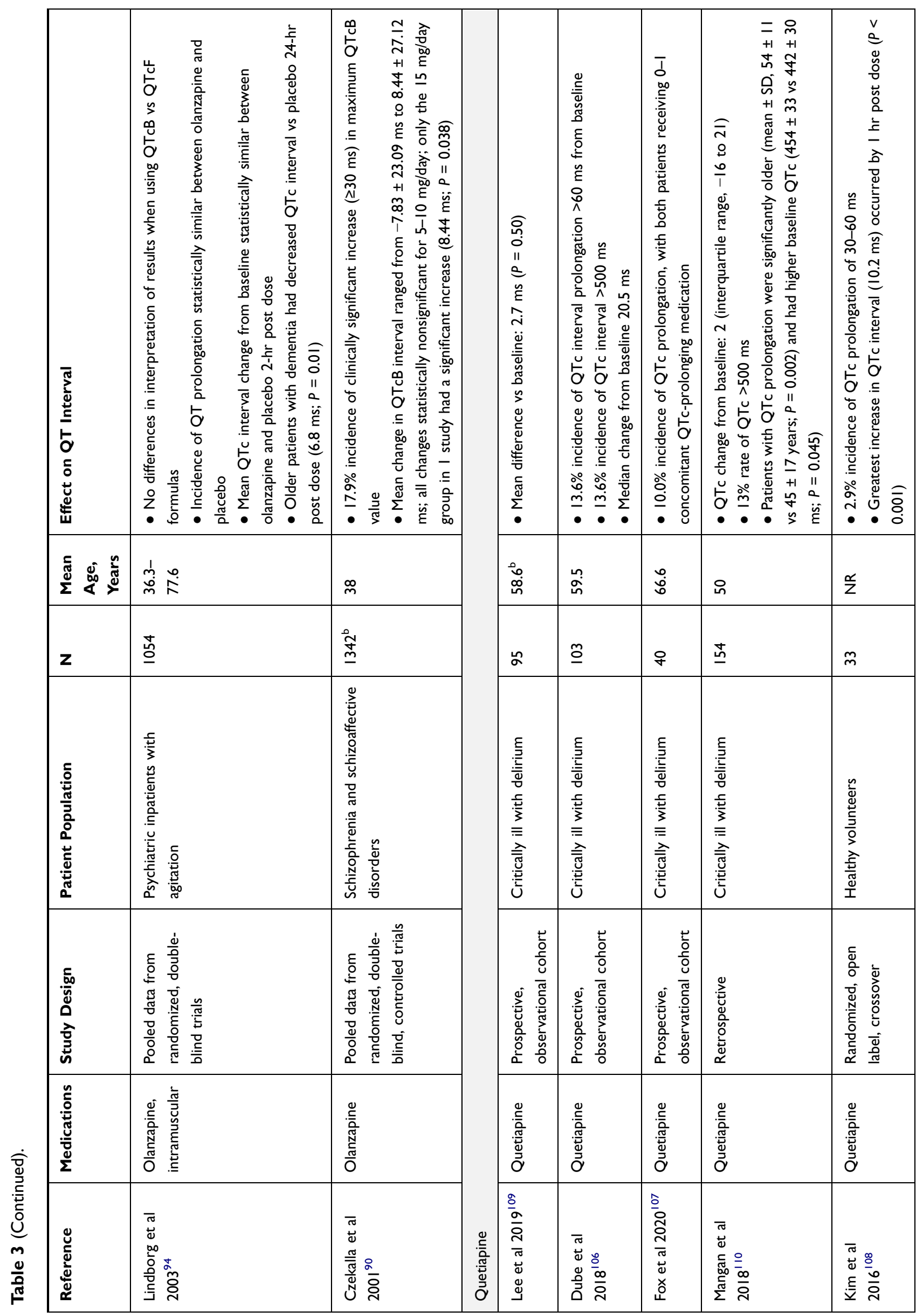




\begin{tabular}{|c|c|c|c|c|c|c|c|}
\hline 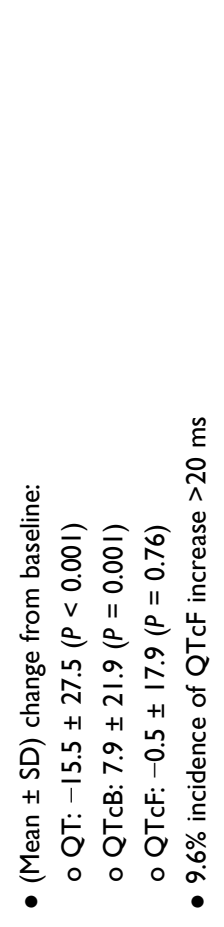 & 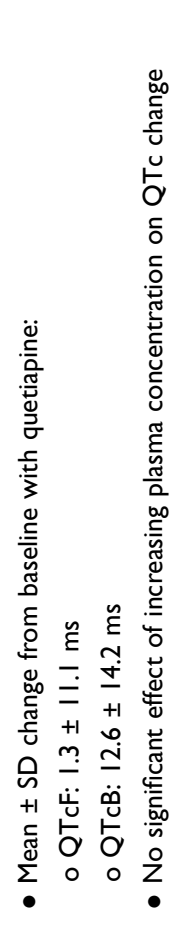 & 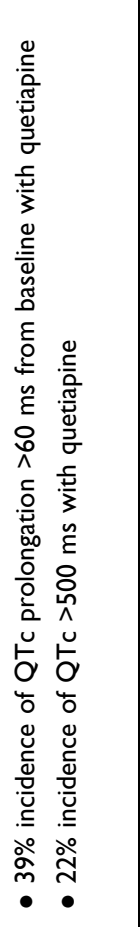 & & 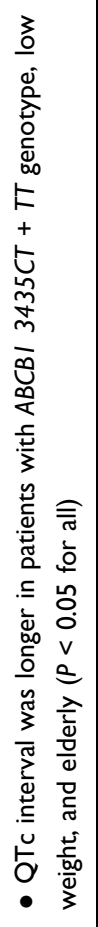 & 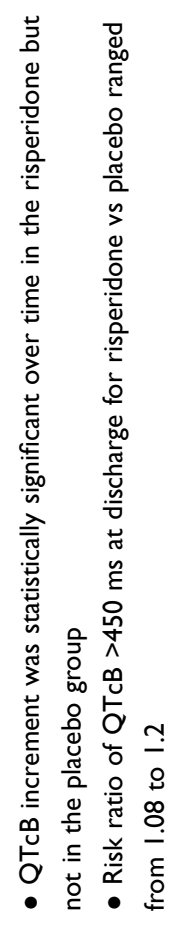 & 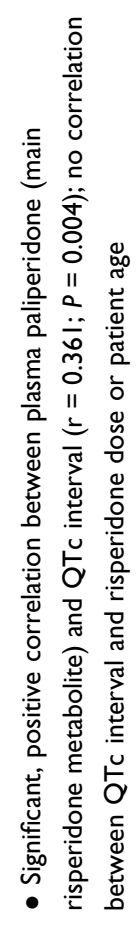 & 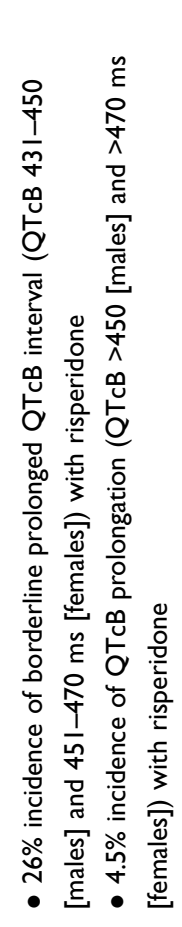 \\
\hline$\stackrel{\mathscr{\alpha}}{z}$ & $\stackrel{\alpha}{z}$ & 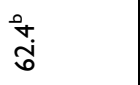 & & $\underset{m}{\stackrel{d}{m}}$ & 茴 & 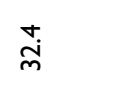 & $\stackrel{\text { L }}{n}$ \\
\hline$\stackrel{\stackrel{g}{ \pm}}{=}$ & $\stackrel{\stackrel{p}{m}}{m}$ & $\stackrel{m}{m}$ & & ஃ & : & $\bar{\sigma}$ & $\stackrel{\circ}{\circ}$ \\
\hline 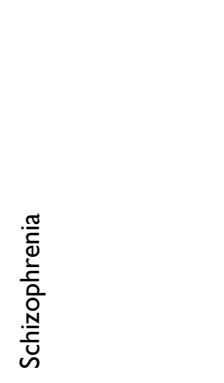 & 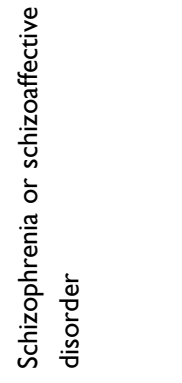 & 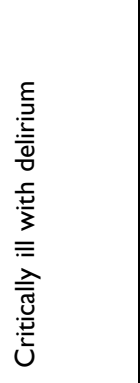 & & 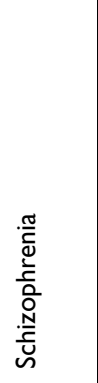 & 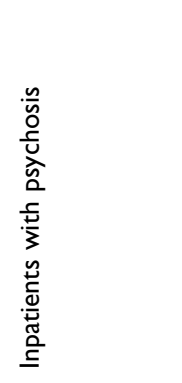 & 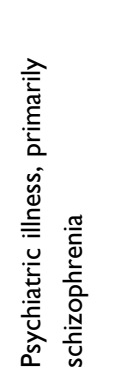 & 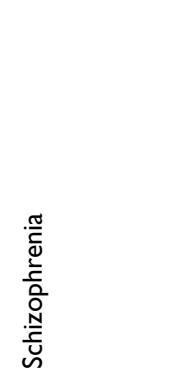 \\
\hline 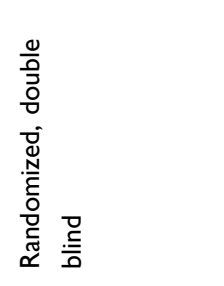 & 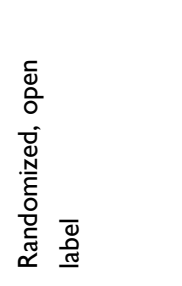 & 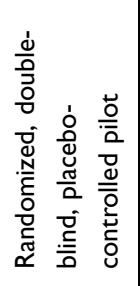 & & 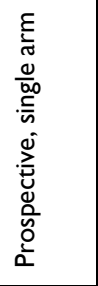 & 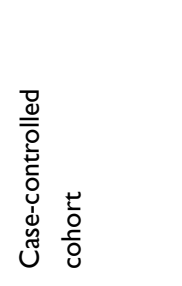 & 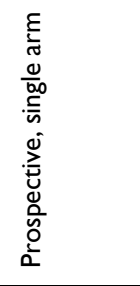 & 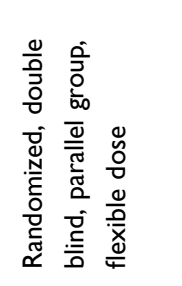 \\
\hline 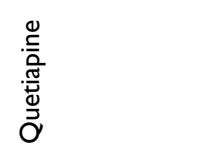 & 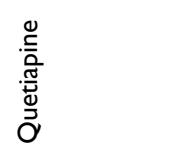 & 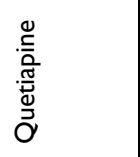 & & 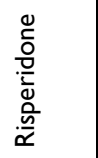 & 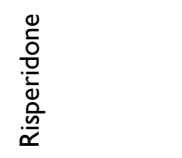 & 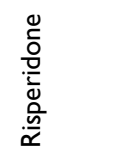 & 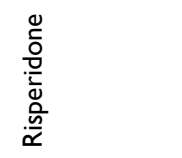 \\
\hline 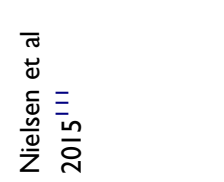 & 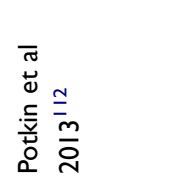 & 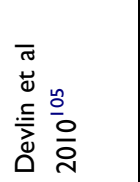 & 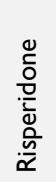 & 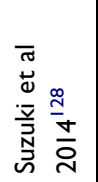 & 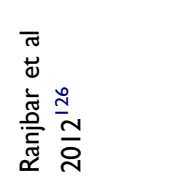 & 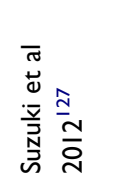 & 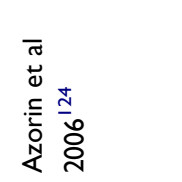 \\
\hline
\end{tabular}




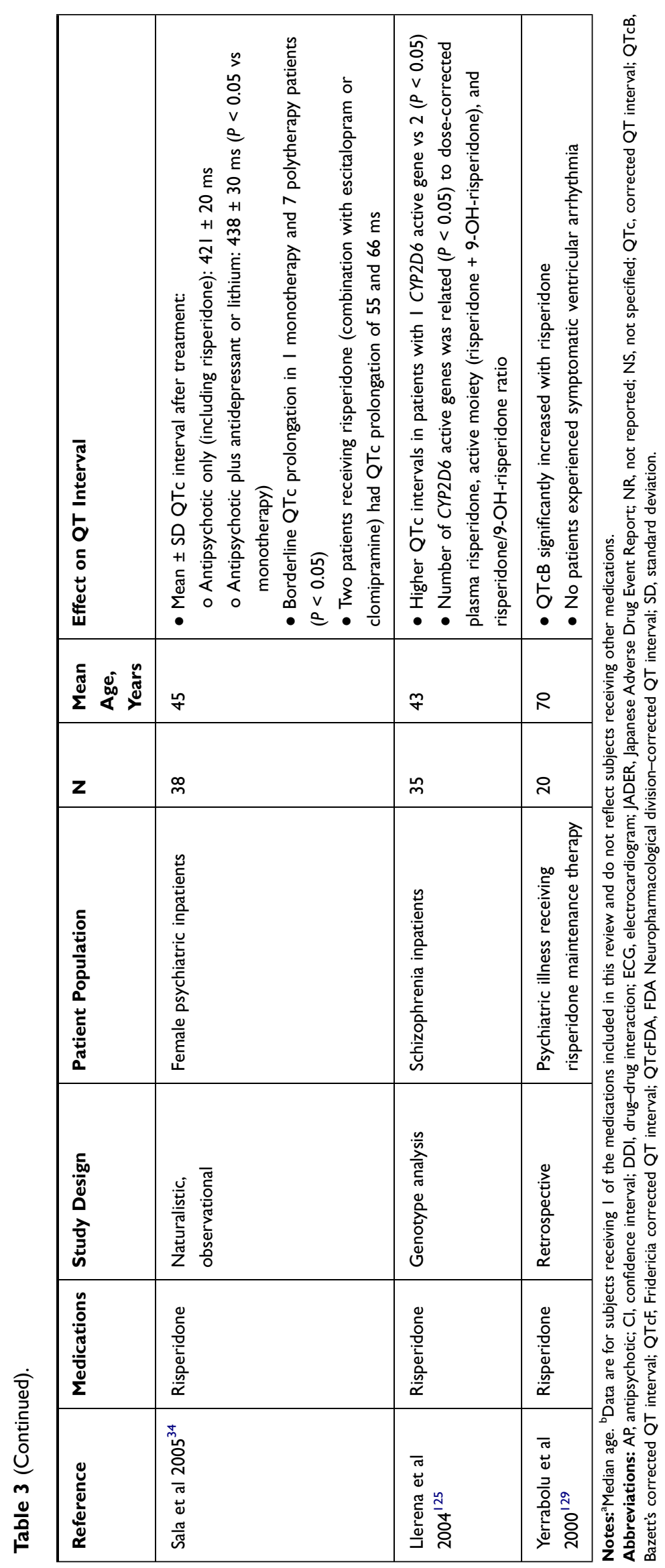




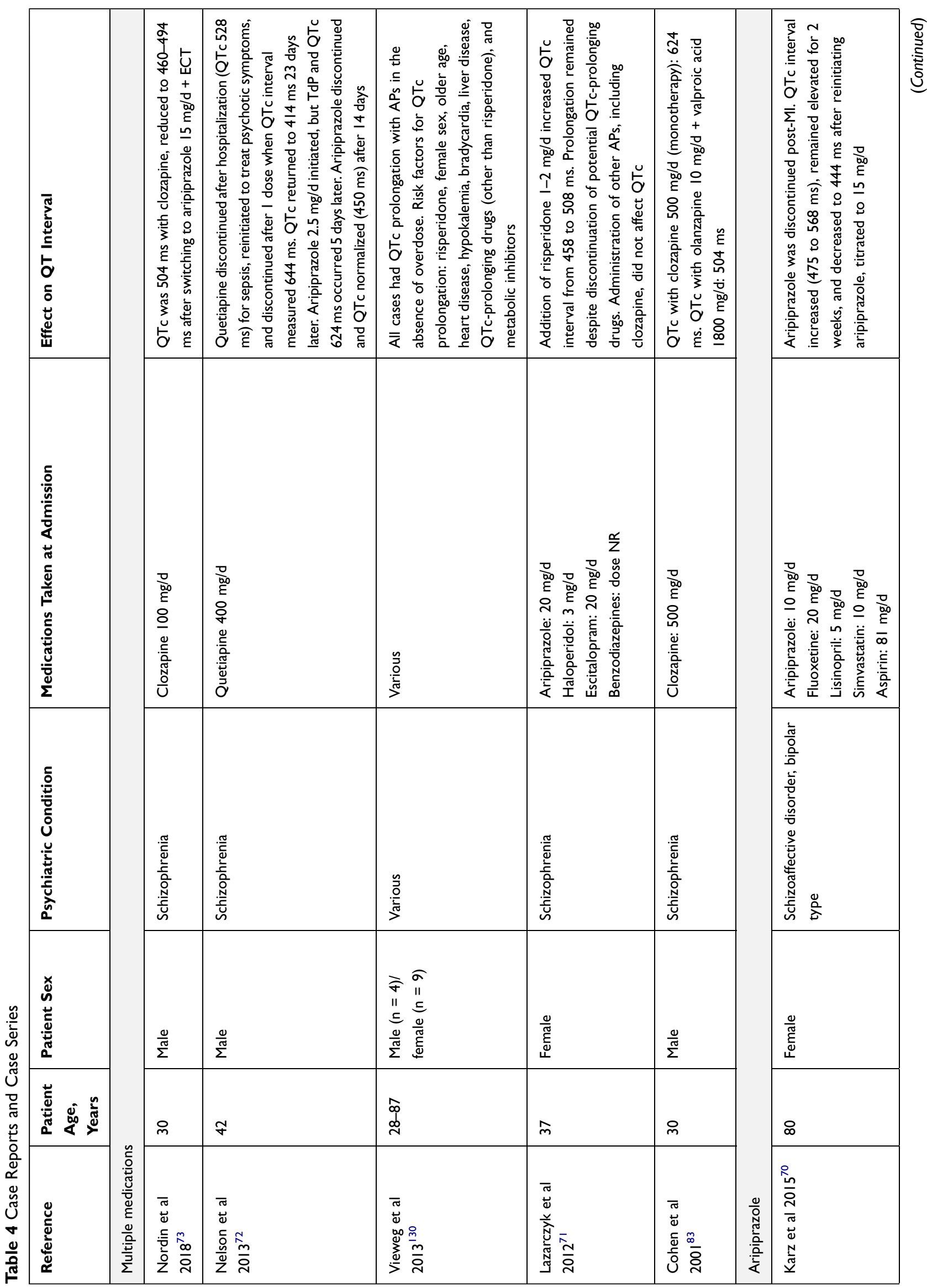




\begin{tabular}{|c|c|c|c|c|c|c|c|c|c|}
\hline 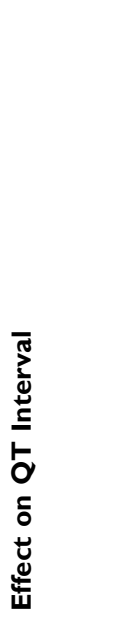 & & 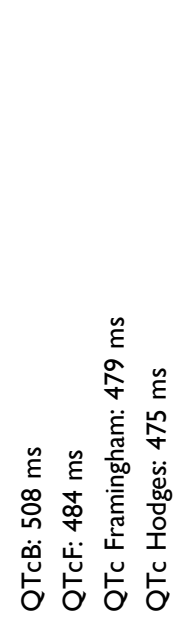 & 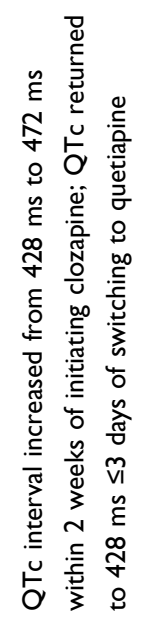 & 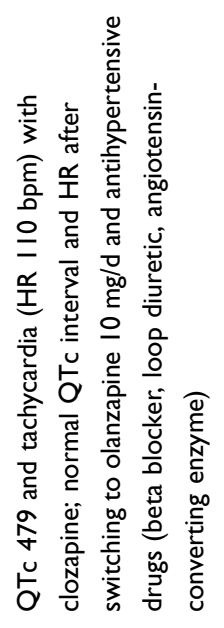 & & 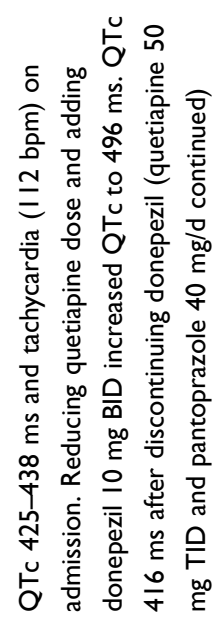 & 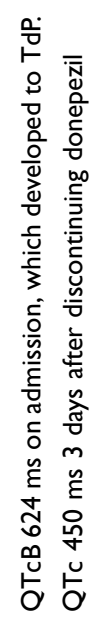 & 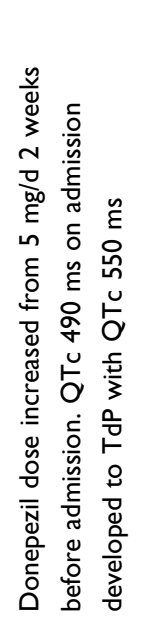 & 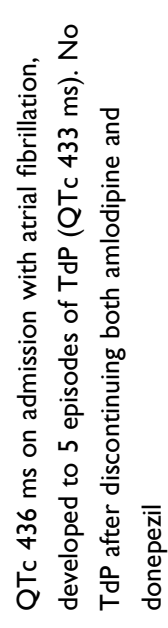 \\
\hline 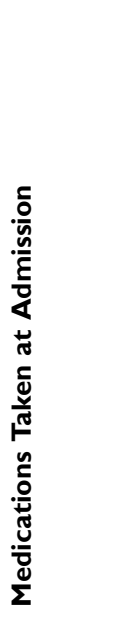 & & 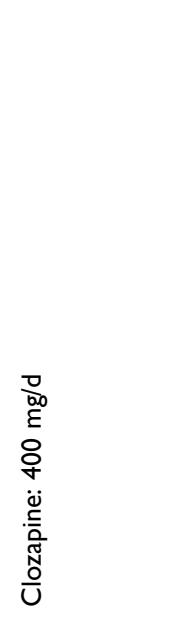 & 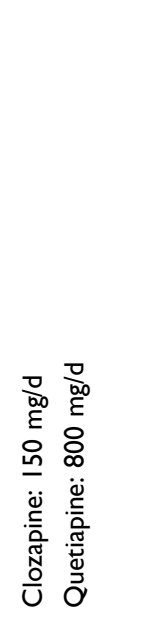 & 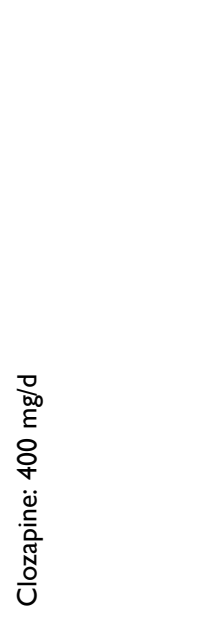 & & 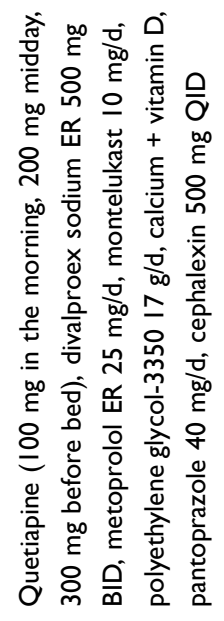 & 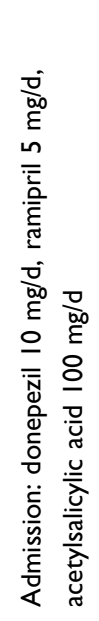 & 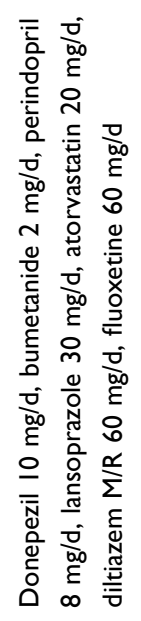 & 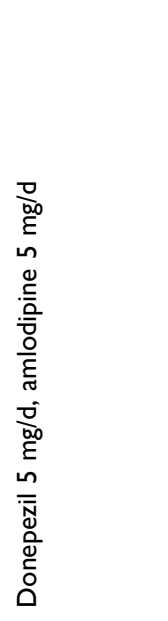 \\
\hline 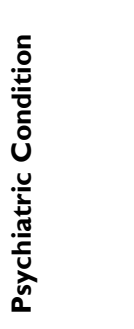 & & 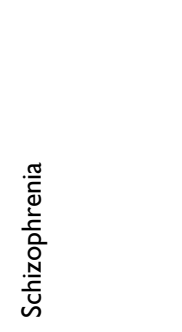 & 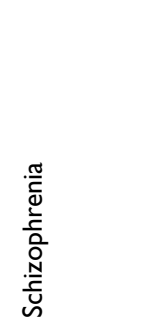 & 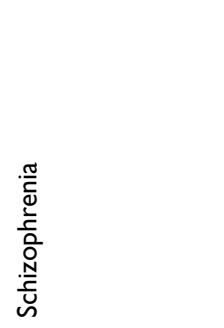 & & 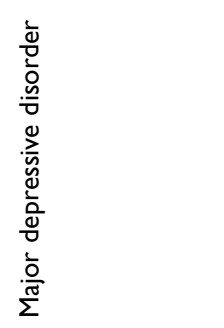 & 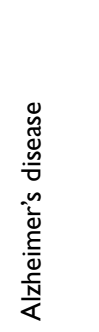 & 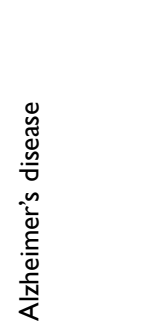 & 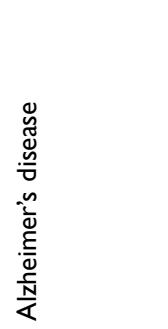 \\
\hline 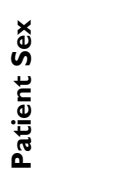 & & $\frac{\frac{\omega}{\pi}}{\sum}$ & $\frac{\frac{\omega}{\pi}}{\sum}$ & $\frac{\frac{\omega}{\tilde{N}}}{\frac{\pi}{\alpha}}$ & & 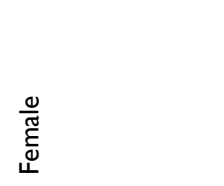 & $\begin{array}{l}\frac{0}{\pi} \\
\frac{\mathscr{\sigma}}{\tilde{W}} \\
\end{array}$ & $\begin{array}{l}\frac{0}{\tilde{\Xi}} \\
\frac{\tilde{E}}{\mathscr{W}}\end{array}$ & $\begin{array}{l}\frac{\mathscr{0}}{\tilde{\Xi}} \\
\frac{\tilde{L}}{L}\end{array}$ \\
\hline 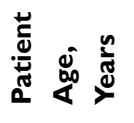 & & $\stackrel{\text { ㅁ }}{*}$ & மே & $\bar{m}$ & & $\stackrel{\sim}{\sim}$ & ఫे & $\infty$ & œ \\
\hline 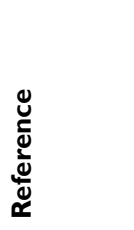 & $\begin{array}{l}\stackrel{0}{\frac{\Xi}{N}} \\
\text { N } \\
\frac{O}{U}\end{array}$ & 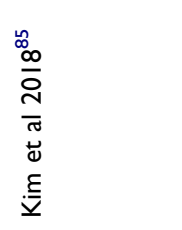 & 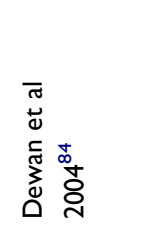 & 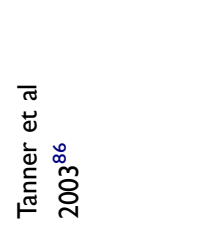 & 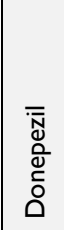 & 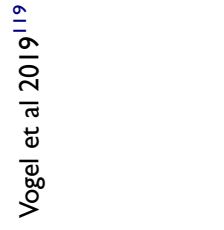 & 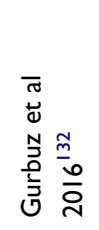 & 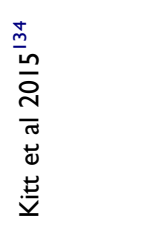 & 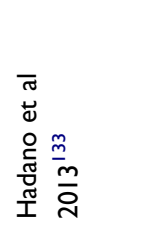 \\
\hline
\end{tabular}




\begin{tabular}{|c|c|c|c|c|c|c|c|}
\hline 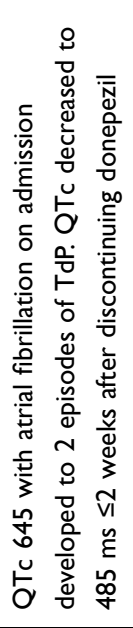 & 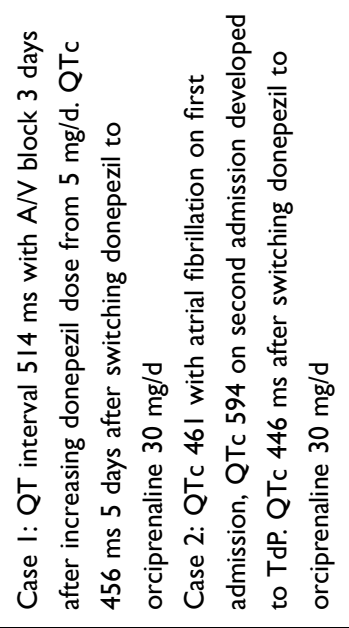 & 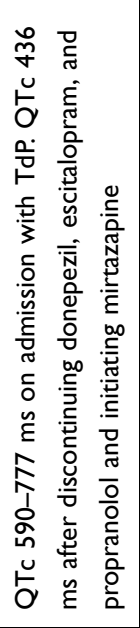 & & 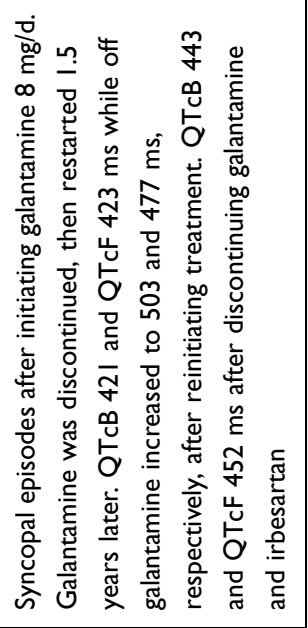 & & 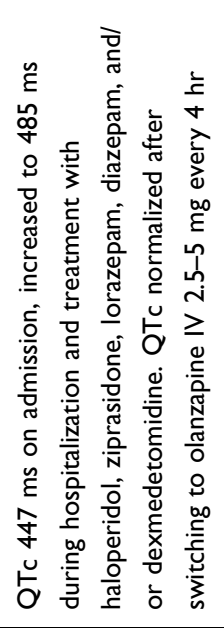 & 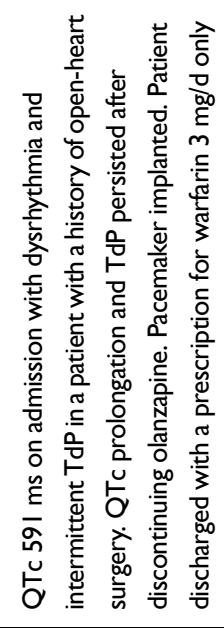 \\
\hline 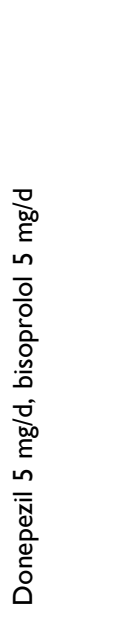 & 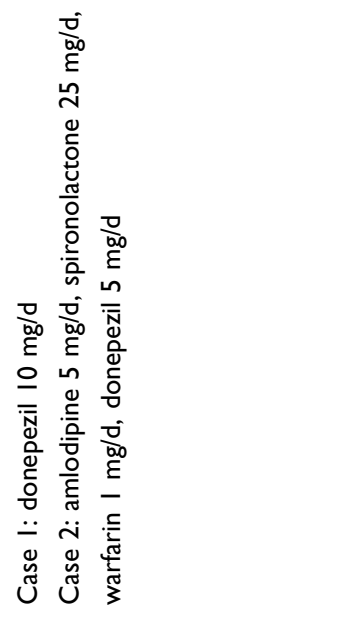 & 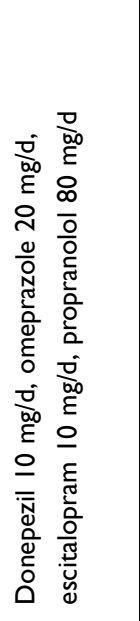 & & 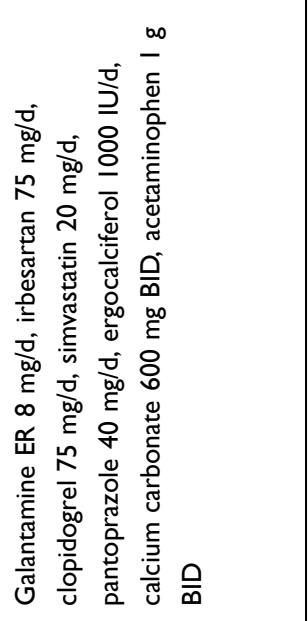 & & 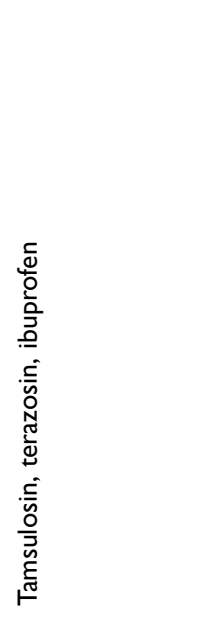 & 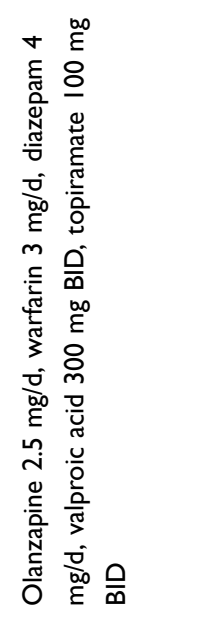 \\
\hline 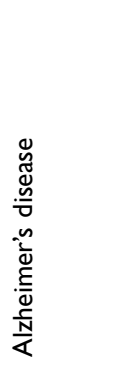 & 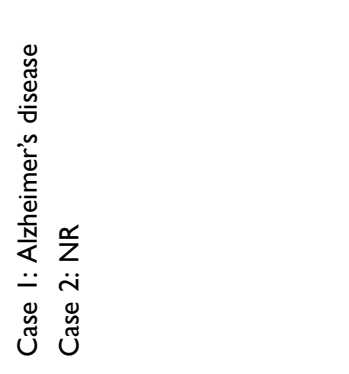 & 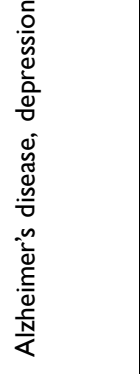 & & 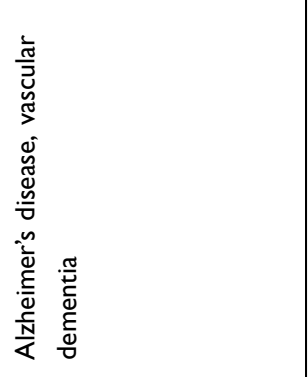 & & 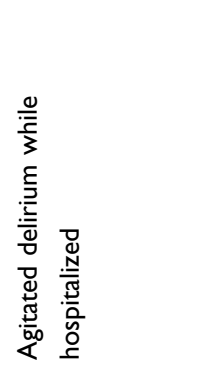 & 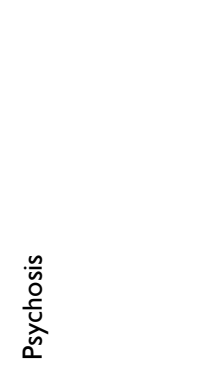 \\
\hline 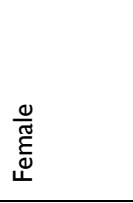 & 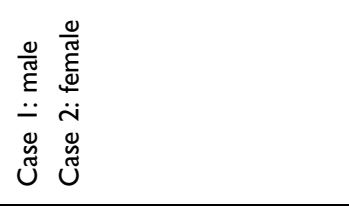 & 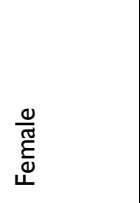 & & $\frac{\frac{\omega}{\pi}}{\frac{\pi}{\pi}}$ & & $\frac{\frac{\omega}{\sigma}}{\Sigma}$ & $\begin{array}{l}\frac{\mathscr{U}}{\tilde{E}} \\
\stackrel{\tilde{\Psi}}{\Psi} \\
\end{array}$ \\
\hline 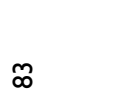 & 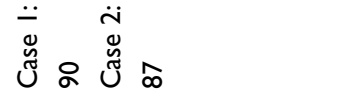 & $\stackrel{2}{\wedge}$ & & $\stackrel{\llcorner}{\infty}$ & & R & $\mathcal{F}$ \\
\hline 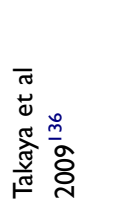 & 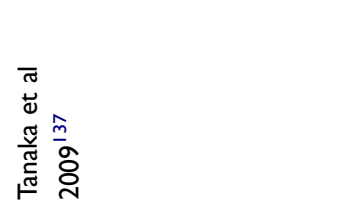 & 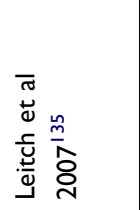 & 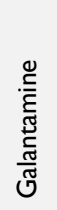 & 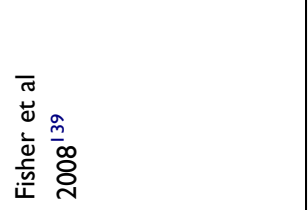 & 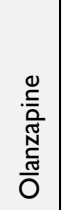 & 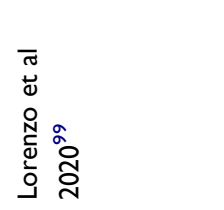 & 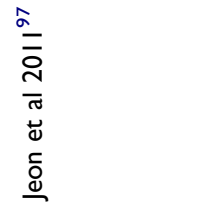 \\
\hline
\end{tabular}




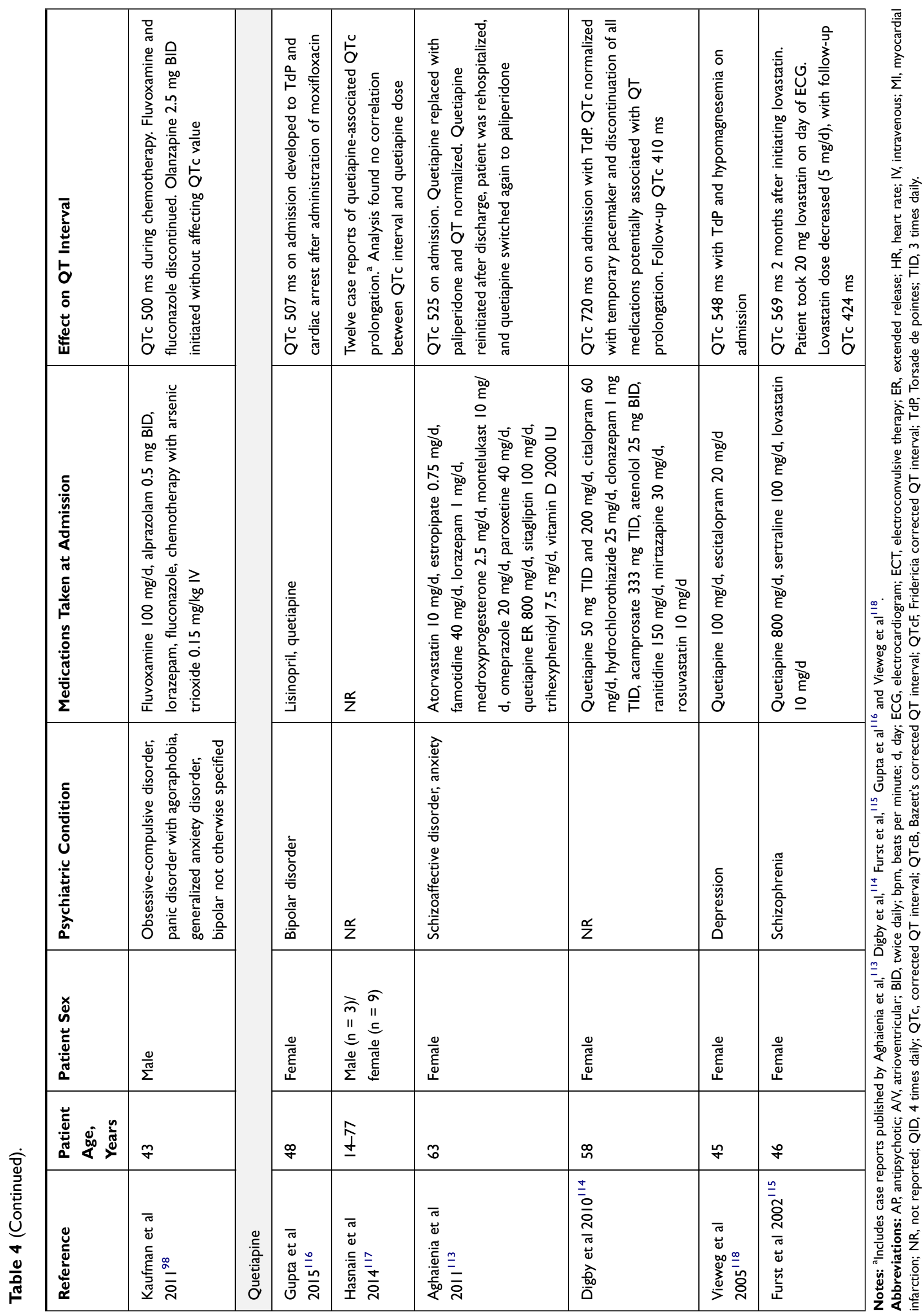


reduced risk of QTc interval prolongation in contrast to other drugs $(P=0.013)$, whereas no significant effect was observed with clozapine, olanzapine, quetiapine, or risperidone. Case reports of patients receiving aripiprazole were consistent with findings from the meta-analyses and clinical studies (Table 4). ${ }^{70,73}$

The product label for aripiprazole lists QT prolongation as a rare event, occurring in fewer than 1 in 1000 patients. ${ }^{21}$ The evidence for QTc interval reduction with aripiprazole, plus its mechanism of action as a dopamine receptor blocker and agonist, would count in its favor as a treatment for PDP. However, aripiprazole is associated with worsened motor function in PD and, therefore, is not recommended for PDP. ${ }^{75,76}$

\section{Clozapine}

The effect of clozapine on QTc interval was described in 1 systematic review, ${ }^{59} 13$ studies, ${ }^{33,45,50,51,55,61,67,77-82}$ and 6 case reports. ${ }^{71,73,83-86}$ One study found no significant change from baseline in mean QTc interval or the incidence of QTc prolongation, ${ }^{79}$ but this is inconsistent with findings from other researchers. Four studies of 4951 psychiatric inpatients have reported an increased risk of QTc prolongation with clozapine (odds ratios, 1.006 [95\% CI, 1.003-1.008] and 2.4 [95\% CI, 1.4-4.2]), with the magnitude of effect being dose dependent (Table 3). ${ }^{45,50,51,82}$

Case reports also reported heterogeneous effects of clozapine. A 37-year-old woman who experienced QTc prolongation (an increase from 458 to $508 \mathrm{~ms}$ ) with risperidone $1-2 \mathrm{mg} /$ day had no such response with clozapine (dose not specified; Table 4). ${ }^{71}$ On the other hand, QTc intervals of 472-504 ms have been reported in men ages $30-45$ years taking clozapine $100-400 \mathrm{mg} /$ day. $^{73,84,86} \mathrm{In}$ each case, QTc returned to baseline after switching to aripiprazole, quetiapine, or olanzapine.

The label for clozapine warns about the risk of QTc prolongation, ${ }^{22}$ but the risk appears to be dose dependent, ${ }^{45,50}$ and patients with PDP require doses 10-fold lower (6.25-50 $\mathrm{mg} /$ day) than those used to treat schizophrenia. ${ }^{6,75}$ However, it is important to keep in mind that because the effect is dosedependent, even a dose that would on its own not cause a significant effect could interact with other QTc-modifying factors (eg, concomitant medication with QTc prolongation effects, age, comorbidities) to cause clinically significant QTc prolongation. $^{50-52}$

Because clozapine does not worsen motor function, it has been used to treat PDP. Long-term treatment with clozapine, however, can cause autonomic dysfunction, including increased heart rate and reduced heart rate variability. Autonomic dysfunction is already a concern in PD, and it further increases the risk of QTc prolongation. ${ }^{77}$ Also, clozapine is associated with agranulocytosis, though the risk is dose dependent and usually avoided in patients with PD if low doses are used. Finally, severe neutropenia (neutrophil count $<0.5 \times 103 / \mu \mathrm{L}$ ) has been reported in $0.91 \%$ of patients treated with clozapine. ${ }^{6,75}$ As a result, weekly blood count monitoring is required during the first year of treatment, which increases the burden for patients and caregivers. ${ }^{6,75}$

\section{Olanzapine}

Olanzapine was mentioned in 6 systematic reviews and metaanalyses, ${ }^{30,31,56,57,87,88} 26$ studies, ${ }^{32,33,47-49,52-55,61,64,67-}$ $69,77,78,80,81,89-96$ and 4 case reports. ${ }^{83,97-99}$ Multiple meta-analyses and clinical studies reported no or minimal effect of oral olanzapine on QTc interval, ${ }^{88,89,91}$ with a placebo-adjusted standardized mean difference of $-0.14 \mathrm{~ms}^{30}$ and $1.7-\mathrm{ms}$ increases from baseline. ${ }^{91}$ Intramuscular olanzapine reduced QT interval by approximately $3 \mathrm{~ms}$ during the 24 hours post injection. ${ }^{30,88,89,94}$ Any QTc effects may be sex-specific: in a study by Suzuki et al, ${ }^{47}$ women who received olanzapine experienced longer mean QTc intervals than men $(P=$ 0.007), whereas such differences were not observed with other antipsychotics (Table 3).

Case reports of patients receiving polypharmacy who had prolonged QTc intervals described no cardiac effects of olanzapine (Table 4). ${ }^{83,97-99}$ To our knowledge, the only exception reported is that of an elderly woman with a QTc prolongation triggered by the addition of the CYP450 inhibitor ciprofloxacin to an established regimen of olanzapine, valsartan, and azathioprine. ${ }^{100}$

The product label for olanzapine does not warn of QTc effects and reports no significant differences in comparison with placebo in the proportions of patients experiencing clinically important QT changes. ${ }^{25}$ Despite the reported cardiac safety of olanzapine, the medication is ineffective in treating psychotic symptoms in PDP and affects motor function; ${ }^{6,75,76}$ it therefore is not recommended in this patient population.

\section{Pimavanserin}

No studies of pimavanserin were identified in our literature search. However, the product label for pimavanserin advises that the medication prolongs the QT interval. ${ }^{20}$ Although clinical trials for pimavanserin were not 
identified in the literature search, QT interval prolongation was mentioned in the publications of these trials. ${ }^{101-104}$ In a Phase 3 study of patients with PDP, pimavanserin was associated with a mean 7.3-ms increase in Bazett's-corrected QTc interval over 6 weeks, in comparison with no change for placebo. ${ }^{102}$ During the open-label extension (OLE) of this study, $12(2.6 \%)$ patients experienced individual events of a Fridericia-corrected QTc interval $>500$ msec, and $6(1.3 \%)$ patients experienced an adverse event of prolonged QT interval. ${ }^{104}$ In the 12-week Phase 2 trial of pimavanserin in patients with Alzheimer's disease, those who received pimavanserin had a mean 9.4-ms increase in Fridericia-corrected QTc interval from baseline, in comparison with a decrease of $2.0 \mathrm{~ms}$ for placebo.${ }^{101}$ One patient in each treatment group had a change from baseline of $\geq 60 \mathrm{~ms}$, recorded at day 15 for both. In the 12 week open-label period of a Phase 3 trial of pimavanserin in patients with dementia-related psychosis, including patients with Parkinson's disease dementia, pimavanserin was associated with a mean Fridericia-corrected QTc prolongation of $5.4 \mathrm{msec}$, with $1(0.3 \%)$ patient experiencing an increase in QTc of $\geq 60 \mathrm{~ms} .{ }^{103}$ In all studies, the effect of pimavanserin on QTc interval was not associated with related adverse events. ${ }^{101-104}$ In 2019, the authors performed a retrospective review of medical records for 48 patients treated with pimavanserin in their movement disorders clinic, with the goal of determining whether their electrocardiograms showed any evidence of QTc abnormalities. No QTc prolongation was identified for any of the 48 patients (data on file).

Pimavanserin is currently the only antipsychotic approved by the US Food and Drug Administration (FDA) for the treatment of hallucinations and delusions associated with PDP. ${ }^{20}$ The product label warns about the increased risk of QTc prolongation. ${ }^{20}$ Pimavanserin is recommended for patients with PDP who do not have preexistent QTc prolongation and are not taking QTcprolonging medications. ${ }^{20}$ A recent OLE safety study demonstrated the long-term safety and tolerability of pimavanserin specifically in patients with PDP, showing that types of adverse events reported in an OLE study were comparable to the 6-week placebo-controlled studies. ${ }^{104}$ Patients were excluded if they received other medications known to prolong the QT interval, or had a baseline Bazett-corrected QT interval of $>460$ for males or $>470$ for females. ${ }^{104}$

\section{Quetiapine}

Quetiapine was mentioned in 4 systematic reviews and meta-analyses, ${ }^{30,31,57,87} 25$ studies, ${ }^{33,47-49,53-55,64,67-}$ $69,78,80,81,91,92,96,105-112$ and 8 publications describing case reports. $^{72,113-119}$ A Cochrane meta-analysis reported no significant difference in risk of QTc prolongation between quetiapine and comparator antipsychotics, but RRs (95\% CIs) favored comparator drugs: aripiprazole, 3.21 (95\% CI, 0.13-76.74); olanzapine, 12.96 (95\% CI, 0.73229.17); and risperidone, 1.34 (95\% CI, 0.36-5.04) (Table 2). ${ }^{87}$ Quetiapine was associated with a $4.81-\mathrm{ms}$ (95\% CI, 0.34-9.28) greater increase in QTc interval than was olanzapine, with similar QTc effects as for risperidone. ${ }^{87}$ The results from individual studies were heterogeneous, with some studies ${ }^{48,111,112}$ showing minimal or no effect on QTc interval and others ${ }^{91,106,108}$ suggesting that quetiapine is associated with mean or median changes from baseline of -5 to $+20 \mathrm{~ms}$ (Table 3 ). A mean change in QTc interval of $-0.5 \pm 17.9 \mathrm{~ms}$ was observed with quetiapine doses of 400-600 mg/day, but in the same study, 9.6\% of patients had increases in QTc interval $>20$ $\mathrm{ms}$ in relation to baseline. ${ }^{111}$ In other studies of psychiatric patients, quetiapine was associated with QTc prolongation of $1.3-5.7 \mathrm{~ms}$ in relation to baseline. ${ }^{69,91,112}$ In healthy volunteers, quetiapine elicited mean QTc interval increases of $10.2 \mathrm{~ms}$, with prolongation occurring in a dose-dependent manner. $^{108}$

The product label for quetiapine states that QTc effects were not observed in clinical trials but postmarketing reports suggest that prolongation can occur in the context of overdose, comorbidity, and coadministration of QTcprolonging medications. ${ }^{29}$ Quetiapine does not appear to worsen extrapyramidal symptoms. However, orthostatic hypotension and somnolence are commonly reported adverse events and may lead to falls because of its properties as an alpha-1 adrenergic and histamine $\mathrm{H} 1$ antagonist. ${ }^{29}$ In addition, randomized controlled trials have not consistently shown efficacy in reducing psychosis symptoms in this patient population. $6,75,76$

\section{Risperidone}

Risperidone was mentioned in 10 systematic reviews and meta-analyses, ${ }^{30,31,56,57,59,87,120-123} 28$ studies, ${ }^{32-34,47-}$ $53,55,60,61,64,67-69,80-82,91,92,124-129$ and 2 case reports. $^{71,130}$ Risperidone generally elicited minimal changes in QTc interval, although interpatient and interstudy variability were observed (Tables 2 and 3). Whereas 1 study 
identified QTc increases from baseline of up to $12 \mathrm{~ms}$ with 3-8 mg/day risperidone, ${ }^{126}$ other studies found no significant effect on QTc interval. ${ }^{32,129}$ A Cochrane meta-analysis reported a greater risk of prolonged QTc intervals in patients receiving risperidone versus placebo. ${ }^{123}$

In healthy individuals, the QTc interval follows a circadian pattern, being slightly prolonged (approximately 5 $\mathrm{ms}$ ) at night in relation to daytime. Risperidone appears to exaggerate this pattern (approximately $14 \mathrm{~ms}$ prolongation), whereas olanzapine does not. ${ }^{52}$

As with olanzapine, the product label for risperidone does not warn of QTc effects and reports no significant differences in comparison with placebo in the proportions of patients experiencing clinically important QT changes. ${ }^{24,25}$ However, risperidone should be avoided for PDP because of the increased risk of extrapyramidal symptoms. ${ }^{6,75,76}$

\section{Dementia Therapies}

A retrospective cohort study of Medicare claims between 2006 and 2014 examined cardiovascular events for patients treated with memantine monotherapy, acetylcholinesterase inhibitor monotherapy (ie, donepezil, rivastigmine, galantamine), or a combination of memantine and acetylcholinesterase inhibitor. ${ }^{131}$ QTc interval prolongation was reported for $0.1 \%-0.2 \%$ of all patients, with no difference between monotherapy and combination therapy. The risk of QTc interval prolongation was also similar between acetylcholinesterase inhibitor and memantine monotherapies and between combination therapy and memantine monotherapy.

\section{Donepezil}

The effect of donepezil on QTc interval was mentioned in 3 studies $^{46,67,131}$ and 7 case reports of 8 patients. ${ }^{119,132-137}$ The prospective studies identified no significant changes from baseline in the QTc intervals of elderly individuals with Alzheimer's disease receiving donepezil 5-10 mg (Table 3). ${ }^{46,131}$ In contrast, a database analysis reported that donepezil, but not antipsychotics or galantamine, was associated with greater odds of long QT syndrome (LQTS) in comparison with other adverse events, with a median 14 days to onset of LQTS. ${ }^{67}$ Seven case reports $(n=8)$ published between 2007 and 2019 described QTc intervals of 463 to $777 \mathrm{~ms},{ }^{119,132-137}$ including 4 cases of TdP and 1 instance of TdP without QTc prolongation (433 ms) (Table 4). ${ }^{132,133,135,136}$ The product label for donepezil does not mention QTc effects in the context of normal (ie, non-overdose) use. ${ }^{6,138}$

\section{Galantamine}

Two studies ${ }^{67,131}$ and 1 case report ${ }^{139}$ described QTc prolongation during galantamine treatment (Table 3). One study identified a $0.2 \%$ rate of QTc prolongation. ${ }^{131}$ In the case report, QTc prolongation (503 ms) occurred in an elderly man who restarted galantamine after a 2-week interruption. $^{139}$ QTc interval had shortened to $443 \mathrm{~ms}$ within 4 days of discontinuing the second round of galantamine (Table 4). Similar to the donepezil label, the galantamine label does not mention effects on QTc during normal use. $^{27}$

\section{Memantine}

Two studies ${ }^{131,140}$ mentioned the effects of memantine on QT interval (Table 3). In the first, 2 (5\%) patients with Alzheimer's disease who received memantine $20 \mathrm{mg} /$ day plus citalopram $30 \mathrm{mg} /$ day experienced QTc interval prolongation, whereas no prolongation was observed in patients who received memantine alone. ${ }^{140}$ The second study showed that $0.1 \%$ of Medicare patients who received memantine experienced QTc interval prolongation. ${ }^{131}$

\section{Rivastigmine}

Only 1 study assessed the effects of rivastigmine on QTc interval, reporting a $0.01 \%$ incidence of QTc prolongation with rivastigmine in patients with Alzheimer's disease (Table 3). ${ }^{131}$ As with other dementia therapies, the product label for rivastigmine does not mention the drug's effects on QTc in the context of normal use. ${ }^{23}$ However, the FDA did not specifically examine QTc prolongation at the time of approval.

\section{Discussion}

Psychosis can occur at any stage of PD and is often treated with antipsychotics or dementia medications. Given the motor and nonmotor features of PD, general patient characteristics (eg, older, with comorbidities), and heterogeneous effects of antipsychotics and dementia therapies, the optimal PDP medication has been unclear. ${ }^{6}$ Dementia therapies are generally preferred for treating cognitive impairment, despite a lack of studies supporting their efficacy for treating hallucinations and delusions associated with PDP. Antipsychotics such as aripiprazole, olanzapine, and risperidone affect motor function and should be avoided in people with PD.

Clinicians must balance the expected benefits with the potential harms when selecting the appropriate medication 
from the remaining options, clozapine, pimavanserin, and quetiapine. For example, clozapine can reduce PDP symptoms but is associated with dose-dependent QTc interval prolongation ${ }^{45,50,51,82}$ and autonomic dysfunction, which is already a concern in PD and further increases the risk of QTc prolongation. ${ }^{77}$ Furthermore, the requirement for ongoing weekly blood draws to monitor for neutropenia and agranulocytosis adds substantial patient and caregiver burden. ${ }^{6,75}$ Quetiapine has not shown efficacy in treating $\mathrm{PDP}^{6,75,76}$ and is not recommended for patients with comorbidities or who receive concomitant QT-prolonging drugs owing to possible prolongation. . $^{291,106,108}$ Quetiapine can also exacerbate underlying fatigue and cause sedation and orthostatic hypotension, all of which may potentially increase the risk of falling. ${ }^{29}$ Pimavanserin was approved by the FDA in April 2016 and is the only medication approved to treat hallucinations and delusions associated with PDP, but it should be avoided in patients with risk factors for prolonged QT interval or who receive QT-prolonging medications. ${ }^{20}$ Pimavanserin has been shown to increase QTc interval by approximately 5-8 $\mathrm{ms}^{20,102}$

As PD progresses, therapeutic options may become increasingly limited due to comorbidities and other medications the patient requires; this may coincide with further development of non-motor symptoms that require treatment. Patients with advanced PD frequently present with multiple risk factors for QTc prolongation or the need for medications that cause QTc prolongation that may interact, such as older age and cardiac comorbidities. In such patients, treatments may include an antipsychotic for psychosis, an acetylcholinesterase inhibitor for apathy, domperidone for orthostatic hypotension, or citalopram for depression. The Movement Disorder Society's (MDS) treatment guidelines caution that citalopram poses a risk for QTc prolongation in older adults, especially at higher doses, and domperidone requires electrocardiogram (ECG) monitoring due to its known association with ventricular tachyarrhythmia and sudden cardiac death in PD patients. ${ }^{141}$ While MDS guidelines state that acetylcholinesterase inhibitors do not require specialized monitoring, they do acknowledge that these medications could affect the patient's ECG, ${ }^{141}$ and the American Geriatrics Society advises caution when combining acetylcholinesterase inhibitors with antipsychotics that affect blood pressure. ${ }^{142}$ It is critical that the physician considers these effects before prescribing these medications to avoid an interaction that produces clinically significant QTc prolongation.
While we pursued a broad review of the literature, some reports may have been excluded due to restrictions that we placed on the search. In particular, conference abstracts can report current analyses that could include data of QTc prolongation with the medications that we examined. Due to the inconsistent and limited availability of conference abstracts and lack of peer review, we considered these abstracts outside of the scope of this review. Additionally, our search terms required mention of "QT interval prolongation," which could have excluded some publications that reported QTc prolongation using alternative terms or phrases such as "QT prolongation" or "arrhythmia."

The magnitudes of QT interval prolongation in the studies included in this review were relatively small (often 5-10 ms), and the clinical importance of small changes in the QT interval has not been determined. QTc prolongation $>500 \mathrm{~ms}$, which has been associated with a risk of $\mathrm{TdP}$, is generally rare with antipsychotics and dementia treatments. ${ }^{54,55}$ The risk of QTc prolongation is increased with the presence of autonomic dysfunction, comorbidities (eg, heart disease, congestive heart failure, myocardial infarction, left ventricular dysfunction, arrhythmia, liver disease, septic shock, thyroid disease), electrolyte imbalance, polypharmacy, increased age, female sex, and genetic variation. . $^{2,51,53,117,122,130,143,144}$ Symptomatic cardiac arrhythmias, congenital QT interval prolongation, hypomagnesemia, and hypokalemia are particular risk factors for further QT prolongation. In the PD population, autonomic dysfunction, comorbidities (eg, heart, liver, or endocrine conditions), polypharmacy, and older age are common, ${ }^{12,145-147}$ and PD occurs at a slightly higher frequency in males than females (1.6:1). ${ }^{148}$

Potential therapies to treat PDP should be considered in the context of other drugs that a patient is receiving for comorbidities to avoid the potential for additive effects of multiple QT-prolonging medications. Drug classes associated with QT interval prolongation include Class 1A or Class 3 antiarrhythmics (eg, quinidine, procainamide, amiodarone, sotalol); antibiotics such as gatifloxacin and moxifloxacin; the antipsychotics ziprasidone, chlorpromazine, and thioridazine; and others (eg, pentamidine, levomethadyl acetate, methadone). ${ }^{20,29}$ Also, because most medications included in this review are metabolized by the CYP450 system, coadministration with CYP450 metabolic inhibitors can theoretically increase plasma concentrations of 1 or more of these agents, increasing the risk for cardiotoxicity. ${ }^{91,100,117,130}$ In clinical studies, metabolic 
inhibitors appear not to influence QTc interval in patients receiving olanzapine, quetiapine, or risperidone, ${ }^{91,112}$ but they can interact with clozapine. ${ }^{22}$ Some authors have also suggested that dose, which is often elevated in patients who require polypharmacy, may play a larger role than polypharmacy in QTc prolongation. ${ }^{33,45,50,51,78}$ Most QTc data from this review were from patients with schizophrenia, who require higher antipsychotic doses than do patients with PDP to alleviate symptoms of psychosis. The use of lower doses for PDP might, therefore, be associated with a reduced risk of QTc prolongation in this patient population.

Clinicians should take care when evaluating QT intervals, to avoid unnecessarily discontinuing useful medications. Although Bazett's formula is commonly used to calculate QTc, it can lead to overcorrection and misunderstanding of the true cardiac effects of medications. ${ }^{149}$ This was demonstrated in a case in which a patient receiving clozapine $400 \mathrm{mg} /$ day had a resting heart rate of 80 beats per minute and QTc values of $508 \mathrm{~ms}$ (Bazett's), $484 \mathrm{~ms}$ (Fridericia), $479 \mathrm{~ms}$ (Framingham), and $475 \mathrm{~ms}$ (Hodges). ${ }^{85}$ Many publications in our review did not report the formula used, and of those that did, Bazett's was more common than Fridericia. In the study by Nielsen et al, ${ }^{111}$ QT intervals were corrected with both Fridericia's and Bazett's formulas. Whereas the Fridericia formula showed a mean QTc change from baseline of $-0.5 \mathrm{~ms} \pm$ $17.9 \mathrm{~ms}$ with quetiapine, Bazett's formula suggested that QTc interval increased by $7.9 \mathrm{~ms} \pm 21.9 \mathrm{~ms}$. Because of the tendency to overcorrect the QT interval, Bazett's formula is not recommended by the American Heart Association for electrocardiogram calculations. ${ }^{149}$

The risk of QT interval prolongation should be evaluated for an individual patient with PD in the context of selecting an appropriate therapy but should not prevent initiating a treatment for hallucinations and delusions. The presence of psychosis in patients with PD has been associated with increased caregiver burden, ${ }^{19}$ nursing home placement, ${ }^{18}$ and mortality, ${ }^{17}$ indicating a need for timely implementation of an effective treatment strategy.

Importantly, none of the publications described in this review were specifically conducted in patients with PDP. Most studies of antipsychotics were done in patients with schizophrenia or other psychiatric disorders, and most studies of antidementia treatments were done in patients with Alzheimer's disease. Though some characteristics may overlap between patients with PDP and patient populations in these studies, risk factors for medication-related cardiac effects may be more common in certain patient populations than in others. For example, increased age and polypharmacy might increase the risk of QTc prolongation in patients with PDP, and these factors may influence treatment decisions. Clinical trials are therefore needed to determine the QTc-prolonging effects of each drug in this unique patient population. Despite the recent requirements set forth by some insurance companies, mandating electrodiagnostic testing before starting a single drug may not be necessary because there is not a clear consensus on the effect of most of these medications on QTc prolongation.

\section{Conclusions}

In general, second-generation antipsychotics are associated with small changes in QTc interval, and pathological QTc prolongation and TdP are rare. The potential for QTc prolongation, however, is important to consider in patients with symptomatic cardiac arrhythmias and those receiving QT-prolonging medications. In choosing a medication to treat PDP, the expected efficacy must be balanced by possible safety concerns for individual patients.

Symptoms of PDP need to be treated and a patient's risk of QT interval prolongation should factor into treatment choice with what is known about medication efficacy and other safety concerns. Pimavanserin is the only FDAapproved treatment for hallucinations and delusions associated with PDP. Clozapine is also effective but can cause (or exacerbate) autonomic dysfunction and requires frequent blood monitoring throughout treatment. The efficacy of quetiapine has not yet been proven in PDP, and other antipsychotics are generally ineffective in PDP or negatively affect motor function. Although clinicians may prescribe acetylcholinesterase inhibitors in the setting of PDP with cognitive impairment, to date, clinical trials have not demonstrated their efficacy in treating specific symptoms of psychosis. Studies are needed that compare the effects of antipsychotics on QT interval in patients with PDP.

\section{Author Contributions}

All authors made a significant contribution to the work reported, whether that is in the conception, study design, execution, acquisition of data, analysis and interpretation, or in all these areas; took part in drafting, revising or critically reviewing the article; gave final approval of the version to be published; have agreed on the journal to which the article has been submitted; and agree to be accountable for all aspects of the work. 


\section{Funding}

Funding for medical writing and editorial support was provided by Acadia Pharmaceuticals Inc. Alison Adams, PhD (Ashfield MedComms, an Ashfield Healthcare company), provided writing support based on input from authors, and Dena McWain (Ashfield MedComms) copyedited and styled the manuscript per journal requirements.

\section{Disclosure}

Yasar Torres-Yaghi serves as speaker and advisor for Acorda Therapeutics Inc., Acadia Pharmaceuticals Inc., Amneal Pharmaceuticals Inc., AbbVie Inc., Abbott Laboratories, Sunovion Pharmaceuticals Inc., and Teva Pharmaceutical Industries Ltd.; advisor for KeifeRx LLC; and speaker for Teva Pharmaceutical Industries Ltd. and US WorldMeds LLC. Amelia Carwin serves as advisor for Acadia Pharmaceuticals Inc. and Kyowa Kirin Co. Ltd. Jacob Carolan has no conflict of interest to report. Steven Nakano serves as advisor for Acadia Pharmaceuticals Inc. and Kyowa Kirin Co. Ltd. Fahd Amjad serves as consultant for Teva, Acadia, Lundbeck, Amneal, AbbVie, Kyowa Kirin, Neurocrine, and Sunovion, outside the submitted work. Fernando Pagan serves as speaker and consultant for Acorda Therapeutics Inc., Acadia Pharmaceuticals Inc., Adamas Pharmaceuticals, Amneal Pharmaceuticals Inc., AbbVie Inc., Abbott Laboratories, Kyowa Kirin Co. Ltd., Merz Pharma GmbH \& Co. KGaA, Sunovion Pharmaceuticals Inc., Teva Pharmaceutical Industries Ltd., and US WorldMeds LLC; is founder of and advisor for KeifeRx LLC; and receives research funding from NIH/NIA, Sun Pharmaceutical Industries Ltd., and Alzheimer's Research Foundation. The authors report no other conflicts of interest in this work.

\section{References}

1. Postuma RB, Berg D, Stern M, et al. MDS clinical diagnostic criteria for Parkinson's disease. Mov Disord. 2015;30(12):1591-1601. doi:10. $1002 / \mathrm{mds} .26424$

2. De Pablo-fernandez E, Tur C, Revesz T, Lees AJ, Holton JL, Warner TT. Association of autonomic dysfunction with disease progression and survival in parkinson disease. JAMA Neurol. 2017;74(8):970-976. doi:10.1001/jamaneurol.2017.1125

3. Grover S, Somaiya M, Kumar S, Avasthi A. Psychiatric aspects of Parkinson's disease. J Neurosci Rural Pract. 2015;6(1):65-76. doi:10.4103/0976-3147.143197

4. Zesiewicz TA, Baker MJ, Wahba M, Hauser RA. Autonomic nervous system dysfunction in Parkinson's disease. Curr Treat Options Neurol. 2003;5(2):149-160. doi:10.1007/s11940-003-0005-0

5. Aarsland D, Creese B, Politis M, et al. Cognitive decline in Parkinson disease. Nat Rev Neurol. 2017;13(4):217-231. doi:10.1038/nrneurol. 2017.27
6. Taddei RN, Cankaya S, Dhaliwal S, Chaudhuri KR. Management of psychosis in Parkinson's disease: emphasizing clinical subtypes and pathophysiological mechanisms of the condition. Parkinsons Dis. 2017;2017:3256542. doi:10.1155/2017/3256542

7. Pagonabarraga J, Martinez-Horta S, Fernández de Bobadilla R, et al. Minor hallucinations occur in drug-naive Parkinson's disease patients, even from the premotor phase. Mov Disord. 2016;31(1):45-52. doi:10.1002/mds.26432

8. Deguchi K, Sasaki I, Tsukaguchi M, et al. Abnormalities of ratecorrected QT intervals in Parkinson's disease - a comparison with multiple system atrophy and progressive supranuclear palsy. $J$ Neurol Sci. 2002;199(1-2):31-37. doi:10.1016/S0022-510X(02) 00079-5

9. Oka H, Mochio S, Sato H, Katayama K. Prolongation of QTc interval in patients with Parkinson's disease. Eur Neurol. 1997;37 (3):186-189. doi:10.1159/000117432

10. Chen Z, Li G, Liu J. Autonomic dysfunction in Parkinson's disease: implications for pathophysiology, diagnosis, and treatment. Neurobiol Dis. 2020;134:104700. doi:10.1016/j.nbd.2019. 104700

11. Orimo S, Takahashi A, Uchihara T, et al. Degeneration of cardiac sympathetic nerve begins in the early disease process of Parkinson's disease. Brain Pathol. 2007;17(1):24-30. doi:10. 1111/j.1750-3639.2006.00032.x

12. Türk AŞ, Köksal A, Altıkka Ö, et al. Assessment of autonomic dysfunction in Parkinson patients by electrocardiogram/Parkinson hastalarında otonomik disfonksiyonun EKG ile değerlendirilmesi. Dusunen Adam. 2012;147-150. doi:10.5350/DAJPN2012250207

13. Gibbons CH, Simon DK, Huang M, et al. Autonomic and electrocardiographic findings in Parkinson's disease. Auton Neurosci. 2017;205:93-98. doi:10.1016/j.autneu.2017.04.002

14. Vandael E, Vandenberk B, Vandenberghe J, Willems R, Foulon V. Risk factors for QTc-prolongation: systematic review of the evidence. Int J Clin Pharm. 2017;39(1):16-25. doi:10.1007/s11096016-0414-2

15. Levin J, Hasan A, Hoglinger GU. Psychosis in Parkinson's disease: identification, prevention and treatment. $J$ Neural Transm (Vienna). 2016;123(1):45-50. doi:10.1007/s00702-015-1400-x

16. Huot P, Johnston TH, Darr T, et al. Increased 5-HT2A receptors in the temporal cortex of parkinsonian patients with visual hallucinations. Mov Disord. 2010;25(10):1399-1408. doi:10.1002/ mds. 23083

17. Forsaa E, Larsen J, Wentzel-Larsen T, Alves G. What predicts mortality in Parkinson disease? A prospective population-based long-term study. Neurology. 2010;75:1270-1276. doi:10.1212/ WNL.0b013e3181f61311

18. Fredericks D, Norton JC, Atchison C, Schoenhaus R, Pill MW. Parkinson's disease and Parkinson's disease psychosis: a perspective on the challenges, treatments, and economic burden. Am J Manag Care. 2017;23:S83-92.

19. Schrag A, Hovris A, Morley D, Quinn N, Jahanshahi M. Caregiverburden in Parkinson's disease is closely associated with psychiatric symptoms, falls, and disability. Parkinsonism Relat Disord. 2006;12 (1):35-41. doi:10.1016/j.parkreldis.2005.06.011

20. Nuplazid (pimavanserin) [prescribing information]. San Diego, CA: Acadia Pharmaceuticals, Inc.; 2020.

21. Abilify (aripiprazole) [prescribing information]. Tokyo, Japan: Otsuka Pharmaceutical Co., Ltd.; 2019.

22. Clozaril (clozapine) [prescribing information]. East Hanover, NJ: Novartis Pharmaceuticals Corp.; 2021.

23. Exelon (rivastigmine) [prescribing information]. East Hanover, NJ: Novartis Pharmaceuticals Corporation; 2018.

24. Risperdal (risperidone) [prescribing information]. Titusville, NJ: Janssen Pharmaceutical Companies; 2021.

25. Zyprexa (olanzapine) [prescribing information]. Indianapollis, IN: Eli Lilly and Company; 2021. 
26. Aricept (donepezil hydrochloride) [prescribing information]. Woodcliff Lake, NJ: Eisai Inc.; 2018.

27. Razadyne ER (galantamine hydrobromide) extended-release [prescribing information]. Titusville, NJ: Janssen Pharmaceuticals, Inc.; 2021.

28. Namenda (memantine $\mathrm{HCl}$ ) [prescribing information]. Madison, NJ; Allergan USA, Inc.; 2018.

29. Seroquel (quetiapine fumarate) [prescribing information]. Wilmington, DE: AstraZeneca Pharmaceuticals LP; 2020.

30. Aronow WS, Shamliyan TA. Effects of atypical antipsychotic drugs on QT interval in patients with mental disorders. Ann Transl Med. 2018;6(8):147. doi:10.21037/atm.2018.03.17

31. Huhn M, Nikolakopoulou A, Schneider-Thoma J, et al. Comparative efficacy and tolerability of 32 oral antipsychotics for the acute treatment of adults with multi-episode schizophrenia: a systematic review and network meta-analysis. Lancet. 2019;394(10202):939-951. doi:10.1016/S0140-6736(19)31135-3

32. Suzuki Y, Sugai T, Ono S, et al. Changes in PR and QTc intervals after switching from olanzapine to risperidone in patients with stable schizophrenia. Psychiatry Clin Neurosci. 2014;68(5):353356. doi:10.1111/pcn.12136

33. Barbui C, Bighelli I, Carra G, et al. Antipsychotic dose mediates the association between polypharmacy and corrected QT interval. PLoS One. 2016;11(2):e0148212. doi:10.1371/journal.pone.0148212

34. Sala M, Vicentini A, Brambilla P, et al. QT interval prolongation related to psychoactive drug treatment: a comparison of monotherapy versus polytherapy. Ann Gen Psychiatry. 2005;4(1):1. doi:10.1186/1744-859X-4-1

35. Alvarez PA, Pahissa J. QT alterations in psychopharmacology: proven candidates and suspects. Curr Drug Saf. 2010;5(1):97104. doi: $10.2174 / 157488610789869265$

36. Bernardi J, Aromolaran KA, Aromolaran AS. Neurological disorders and risk of arrhythmia. Int J Mol Sci. 2020;22(1):188 doi:10.3390/ijms 22010188

37. Huang XP, Mangano T, Hufeisen S, Setola V, Roth BL. Identification of human Ether-à-go-go related gene modulators by three screening platforms in an academic drug-discovery setting. Assay Drug Dev Technol. 2010;8(6):727-742. doi:10.1089/ adt.2010.0331

38. Park J, Cho K-H, Lee HJ, Choi J-S, Rhie D-J. Open channel block of Kv1.4 potassium channels by aripiprazole. Korean J Physiol Pharmacol. 2020;24(6):545-553. doi:10.4196/kjpp.2020.24.6.545

39. ACADIA Pharmaceuticals. NUPLAZID (pimavanserin). Sponsor background information for a meeting of the psychopharmacologic drugs advisory committee on 29 March 2016; 2016. Available from: https://www.fda.gov/media/96755/download. Accessed August 27, 2021.

40. Malik BH, Hamid P, Khan S, Gupta D, Islam M. Correlation between donepezil and QTc prolongation and torsades de pointes: a very rare phenomenon. Cureus. 2019;11(12):e6451.

41. Liu Y, Xu X, Zhang Y, et al. Thioridazine induces cardiotoxicity via reactive oxygen species-mediated hERG channel deficiency and L-type calcium channel activation. Oxid Med Cell Longev. 2020;2020:3690123. doi:10.1155/2020/3690123

42. Abbaszadeh S, Javidmehr A, Askari B, Janssen PML, Soraya H. Memantine, an NMDA receptor antagonist, attenuates cardiac remodeling, lipid peroxidation and neutrophil recruitment in heart failure: a cardioprotective agent? Biomed Pharmacother. 2018;108:1237-1243. doi:10.1016/j.biopha.2018.09.153

43. Gallini A, Sommet A, Montastruc J-L. Does memantine induce bradycardia? A study in the French PharmacoVigilance Database. Pharmacoepidemiol Drug Saf. 2008;17(9):877-881. doi:10.1002/ pds. 1620

44. Takehara H, Suzuki Y, Someya T. QT prolongation associated with memantine in Alzheimer's disease. Psychiatr Clin Neurosci. 2015;69(4):239-240. doi:10.1111/pcn.12236
45. Kang UG, Kwon JS, Ahn YM, et al. Electrocardiographic abnormalities in patients treated with clozapine. $J$ Clin Psychiatry. 2000;61(6):441-446. doi:10.4088/JCP.v61n0609

46. Isik AT, Yildiz GB, Bozoglu E, Yay A, Aydemir E. Cardiac safety of donepezil in elderly patients with Alzheimer disease. Intern Med. 2012;51(6):575-578. doi:10.2169/internalmedicine.51.6671

47. Suzuki Y, Sugai T, Fukui N, et al. Sex differences in the effect of four second-generation antipsychotics on QTc interval in patients with schizophrenia. Hum Psychopharmacol. 2013;28(3):215-219. doi:10.1002/hup.2309

48. Ozeki Y, Fujii K, Kurimoto N, et al. QTc prolongation and antipsychotic medications in a sample of 1017 patients with schizophrenia. Prog Neuropsychopharmacol Biol Psychiatry. 2010;34(2):401-405. doi:10.1016/j.pnpbp.2010.01.008

49. Olsen RE, Kroken RA, Bjorhovde S, et al. Influence of different second generation antipsychotics on the QTc interval: a pragmatic study. World J Psychiatr. 2016;6(4):442-448. doi:10.5498/wjp.v6. i4.442

50. Lin CH, Chen MC, Wang SY, Lin CY. Predictive factors for QTc prolongation in schizophrenic patients taking antipsychotics. $J$ Formos Med Assoc. 2004;103(6):437-441.

51. Yang FD, Wang XQ, Liu XP, et al. Sex difference in QTc prolongation in chronic institutionalized patients with schizophrenia on long-term treatment with typical and atypical antipsychotics. Psychopharmacology (Berl). 2011;216(1):9-16. doi:10.1007/ s00213-011-2188-5

52. Watanabe J, Suzuki Y, Fukui N, et al. Increased risk of antipsychotic-related QT prolongation during nighttime: a 24-hour holter electrocardiogram recording study. J Clin Psychopharmacol. 2012;32(1):18-22. doi:10.1097/JCP.0b013e31823f6f21

53. Aberg K, Adkins DE, Liu Y, et al. Genome-wide association study of antipsychotic-induced QTc interval prolongation. Pharmacogenomics J. 2012;12(2):165-172. doi:10.1038/tpj.2010.76

54. Meyer-Massetti C, Vaerini S, Ratz Bravo AE, Meier CR, Guglielmo BJ. Comparative safety of antipsychotics in the WHO pharmacovigilance database: the haloperidol case. Int J Clin Pharm. 2011;33 (5):806-814. doi:10.1007/s11096-011-9541-y

55. Friedrich ME, Winkler D, Konstantinidis A, et al. Cardiovascular adverse reactions during antipsychotic treatment: results of AMSP, a drug surveillance program between 1993 and 2013. Int $J$ Neuropsychopharmacol. 2020;23(2):67-75. doi:10.1093/ijnp/pyz046

56. Chung AK, Chua SE. Effects on prolongation of Bazett's corrected QT interval of seven second-generation antipsychotics in the treatment of schizophrenia: a meta-analysis. J Psychopharmacol. 2011;25 (5):646-666. doi:10.1177/0269881110376685

57. Leucht S, Cipriani A, Spineli L, et al. Comparative efficacy and tolerability of 15 antipsychotic drugs in schizophrenia: a multipletreatments meta-analysis. Lancet. 2013;382(9896):951-962. doi:10.1016/S0140-6736(13)60733-3

58. Polcwiartek C, Sneider B, Graff C, et al. The cardiac safety of aripiprazole treatment in patients at high risk for torsade: a systematic review with a meta-analytic approach. Psychopharmacology (Berl). 2015;232(18):3297-3308. doi:10.1007/s00213-015-4024-9

59. Takeuchi H, Suzuki T, Remington G, Uchida H. Antipsychotic polypharmacy and corrected QT interval: a systematic review. Can $J$ Psychiatry. 2015;60(5):215-222. doi:10.1177/0706743715060 00503

60. Chan HY, Lin WW, Lin SK, et al. Efficacy and safety of aripiprazole in the acute treatment of schizophrenia in Chinese patients with risperidone as an active control: a randomized trial. J Clin Psychiatry. 2007;68(1):29-36. doi:10.4088/JCP. v68n0104

61. Hatta K, Hasegawa H, Imai A, et al. Real-world effectiveness of antipsychotic monotherapy and polytherapy in 1543 patients with acute-phase schizophrenia. Asian J Psychiatr. 2019;40:82-87. doi:10.1016/j.ajp.2019.02.005 
62. Kane JM, Carson WH, Saha AR, et al. Efficacy and safety of aripiprazole and haloperidol versus placebo in patients with schizophrenia and schizoaffective disorder. $J$ Clin Psychiatry. 2002;63(9):763-771. doi:10.4088/JCP.v63n0903

63. Keck PE, Marcus R, Tourkodimitris S, et al. A placebo-controlled, double-blind study of the efficacy and safety of aripiprazole in patients with acute bipolar mania. Am J Psychiatry. 2003;160(9):1651-1658. doi:10.1176/appi.ajp.160.9.1651

64. Kram BL, Kram SJ, Brooks KR. Implications of atypical antipsychotic prescribing in the intensive care unit. J Crit Care. 2015;30(4):814-818. doi:10.1016/j.jcrc.2015.03.030

65. Madhusoodanan S, Brenner R, Gupta S, Reddy H, Bogunovic O. Clinical experience with aripiprazole treatment in ten elderly patients with schizophrenia or schizoaffective disorder: retrospective case studies. CNS Spectr. 2004;9(11):862-867. doi:10.1017/ S1092852900002273

66. Pigott TA, Carson WH, Saha AR, et al. Aripiprazole for the prevention of relapse in stabilized patients with chronic schizophrenia: a placebo-controlled 26-week study. J Clin Psychiatry. 2003;64(9):1048-1056. doi:10.4088/JCP.v64n0910

67. Sasaoka S, Matsui T, Hane Y, et al. Time-to-onset analysis of drug-induced long QT syndrome based on a spontaneous reporting system for adverse drug events. PLoS One. 2016;11(10): e0164309. doi:10.1371/journal.pone.0164309

68. Spellmann I, Reinhard MA, Veverka D, et al. QTc prolongation in short-term treatment of schizophrenia patients: effects of different antipsychotics and genetic factors. Eur Arch Psychiatry Clin Neurosci. 2018;268(4):383-390. doi:10.1007/s00406-018-0880-8

69. Suzuki Y, Sugai T, Fukui N, et al. Changes in QT interval after switching to quetiapine in Japanese patients with schizophrenia. Hum Psychopharmacol. 2013;28(1):94-96. doi:10.1002/hup.2277

70. Karz AJ, McGonigle DP, Goldberg JF, Kellner CH, Calenda BS. Effects of aripiprazole on the QTc: a case report. $J$ Clin Psychiatry. 2015;76(12):1648-1649. doi:10.4088/JCP.14cr09539

71. Lazarczyk MJ, Bhuiyan ZA, Perrin N, Giannakopoulos P. Selective acquired long QT syndrome (saLQTS) upon risperidone treatment. BMC Psychiatr. 2012;12:220. doi:10.1186/1471-244X-12-220

72. Nelson S, Leung JG. Torsades de pointes after administration of low-dose aripiprazole. Ann Pharmacother. 2013;47(2):e11. doi:10.1345/aph.1R387

73. Nordin MF, Othman Z. Combined aripiprazole and electroconvulsive therapy in a patient with treatment-resistant schizophrenia and QT prolongation. Int Med J. 2018;25(5):291-292.

74. Sachs G, Sanchez R, Marcus R, et al. Aripiprazole in the treatment of acute manic or mixed episodes in patients with bipolar I disorder: a 3-week placebo-controlled study. J Psychopharmacol. 2006;20(4):536-546. doi:10.1177/0269881106059693

75. Divac N, Stojanovic R, Savic Vujovic K, Medic B, Damjanovic A, Prostran M. The efficacy and safety of antipsychotic medications in the treatment of psychosis in patients with Parkinson's disease. Behav Neurol. 2016;2016:4938154. doi:10.1155/2016/4938154

76. Friedman JH. Parkinson disease psychosis: update. Behav Neurol. 2013;27(4):469-477. doi:10.1155/2013/645429

77. Cohen H, Loewenthal U, Matar M, Kotler M. Association of autonomic dysfunction and clozapine. Heart rate variability and risk for sudden death in patients with schizophrenia on long-term psychotropic medication. Br J Psychiatry. 2001;179:167-171. doi:10.1192/bjp.179.2.167

78. Correll CU, Frederickson AM, Figen V, et al. The QTc interval and its dispersion in patients receiving two atypical antipsychotics. Eur Arch Psychiatry Clin Neurosci. 2009;259(1):23-27. doi:10.1007/s00406-008-0829-4

79. Grande I, Pons A, Baeza I, Torras A, Bernardo M. QTc prolongation: is clozapine safe? Study of 82 cases before and after clozapine treatment. Hum Psychopharmacol. 2011;26(6):397-403. doi:10.1002/hup.1221
80. Rodriguez-Leal CM, Lopez-Lunar E, Carrascosa-Bernaldez JM, Provencio-Arranz RM. Electrocardiographic surveillance in a psychiatric institution: avoiding iatrogenic cardiovascular death. Int J Psychiatry Clin Pract. 2017;21(1):64-66. doi:10.1080/ 13651501.2016.1234623

81. Tumuklu MN, Tumuklu MM, Nesterenko V, Jayathilake K, Beasley CM, Meltzer HY. Twenty-four-hour measures of heart rate-corrected QT interval, peak-to-end of the T-wave, and peakto-end of the T-wave/corrected QT interval ratio during antipsychotic treatment. J Clin Psychopharmacol. 2019;39(2):100-107. doi:10.1097/JCP.0000000000001003

82. Xiang YT, Chiu HF, Ungvari GS, et al. QTc prolongation in schizophrenia patients in Asia: clinical correlates and trends between 2004 and 2008/2009. Hum Psychopharmacol. 2015;30 (2):94-99. doi:10.1002/hup.2458

83. Cohen H, Loewenthal U, Matar MA, Kotler M. Reversal of pathologic cardiac parameters after transition from clozapine to olanzapine treatment: a case report. Clin Neuropharmacol. 2001;24(2):106-108. doi:10.1097/0000282 6-200103000-00008

84. Dewan V, Roth BA. Antipsychotic-induced QTc interval prolongation. Can J Psychiatry. 2004;49(9):646. doi:10.1177/07067 4370404900923

85. Kim DD, White RF, Barr AM, Honer WG, Procyshyn RM. Clozapine, elevated heart rate and QTc prolongation. $J$ Psychiatry Neurosci. 2018;43(1):71-72. doi:10.1503/jpn.170135

86. Tanner MA, Culling W. Clozapine associated dilated cardiomyopathy. Postgrad Med J. 2003;79(933):412-413. doi:10.1136/ pmj.79.933.412

87. Asmal L, Flegar SJ, Wang J, Rummel-Kluge C, Komossa K, Leucht S. Quetiapine versus other atypical antipsychotics for schizophrenia. Cochrane Database Syst Rev. 2013;(11):CD00 6625. doi:10.1002/14651858.CD006625.pub3

88. Kishi T, Matsunaga S, Iwata N. Intramuscular olanzapine for agitated patients: a systematic review and meta-analysis of randomized controlled trials. J Psychiatr Res. 2015;68:198-209. doi:10.1016/j.jpsychires.2015.07.005

89. Brown RR, Estoup MW. Comparison of the metabolic effects observed in patients treated with ziprasidone versus olanzapine. Int Clin Psychopharmacol. 2005;20(2):105-112. doi:10.1097/ 00004850-200503000-00008

90. Czekalla J, Beasley CM, Dellva MA, Berg PH, Grundy S. Analysis of the QTc interval during olanzapine treatment of patients with schizophrenia and related psychosis. J Clin Psychiatry. 2001;62(3):191-198. doi:10.4088/JCP.v62n0310

91. Harrigan EP, Miceli JJ, Anziano R, et al. A randomized evaluation of the effects of six antipsychotic agents on QTc, in the absence and presence of metabolic inhibition. $J$ Clin Psychopharmacol. 2004;24(1):62-69. doi:10.1097/01.jcp.00001 04913.75206 .62

92. Khan Q, Ismail M, Haider I, Khan F. Prevalence of QT interval prolonging drug-drug interactions (QT-DDIs) in psychiatry wards of tertiary care hospitals in Pakistan: a multicenter cross-sectional study. Int J Clin Pharm. 2017;39(6):1256-1264. doi:10.1007/ s11096-017-0532-5

93. Kwon JS, Mittoux A, Hwang JY, Ong A, Cai ZJ, Su TP. The efficacy and safety of 12 weeks of treatment with sertindole or olanzapine in patients with chronic schizophrenia who did not respond successfully to their previous treatments: a randomized, double-blind, parallel-group, flexible-dose study. Int Clin Psychopharmacol. 2012;27(6):326-335. doi:10.1097/YIC.0b013 e32835767a0

94. Lindborg SR, Beasley CM, Alaka K, Taylor CC. Effects of intramuscular olanzapine vs. haloperidol and placebo on QTc intervals in acutely agitated patients. Psychiatry Res. 2003;119 (1-2):113-123. doi:10.1016/S0165-1781(03)00107-0 
95. Petersen AB, Andersen SE, Christensen M, Larsen HL. Adverse effects associated with high-dose olanzapine therapy in patients admitted to inpatient psychiatric care. Clin Toxicol (Phila). 2014;52(1):39-43. doi:10.3109/15563650.2013.862258

96. Viscogliosi G, Chiriac IM, Ettorre E. Efficacy and safety of citalopram compared to atypical antipsychotics on agitation in nursing home residents with alzheimer dementia. $J$ Am Med Dir Assoc. 2017;18(9):799-802. doi:10.1016/j.jamda.2017.06.010

97. Jeon JH, Her SH, Chin JY, et al. Complete atrioventricular blockinduced Torsade de pointes, manifested by epilepsy. Korean $J$ Intern Med. 2011;26(1):99-102. doi:10.3904/kjim.2011.26.1.99

98. Kaufman KR, Chhabra S, Levitt M, Sood R. Arsenic trioxide and olanzapine co-administration: case analysis. J Oncol Pharm Pract. 2011;17(3):260-264. doi:10.1177/1078155209352912

99. Lorenzo MP, Burgess J, Darko W. Intravenous olanzapine in a critically ill patient: an evolving route of administration. Hosp Pharm. 2020;55(2):108-111. doi:10.1177/0018578718823484

100. Letsas KP, Sideris A, Kounas SP, Efremidis M, Korantzopoulos P, Kardaras F. Drug-induced QT interval prolongation after ciprofloxacin administration in a patient receiving olanzapine. Int $J$ Cardiol. 2006;109(2):273-274. doi:10.1016/j.ijcard.2005.04.031

101. Ballard C, Banister C, Khan Z, et al. Evaluation of the safety, tolerability, and efficacy of pimavanserin versus placebo in patients with Alzheimer's disease psychosis: a phase 2, randomised, placebo-controlled, double-blind study. Lancet Neurol. 2018;17(3):213-222. doi:10.1016/S1474-4422(18)30039-5

102. Cummings J, Isaacson S, Mills R, et al. Pimavanserin for patients with Parkinson's disease psychosis: a randomised, placebo-controlled phase 3 trial. Lancet. 2014;383(9916):533-540. doi:10.1016/S0140-6736(13)62106-6

103. Tariot PN, Cummings JL, Soto-Martin ME, et al. Trial of pimavanserin in dementia-related psychosis. $N$ Engl J Med. 2021;385 (4):309-319. doi:10.1056/NEJMoa2034634

104. Ballard CG, Kreitzman DL, Isaacson S, et al. Long-term evaluation of open-label pimavanserin safety and tolerability in Parkinson's disease psychosis. Parkinsonism Relat Disord. 2020;77:100-106. doi:10.1016/j.parkreldis.2020.06.026

105. Devlin JW, Roberts RJ, Fong JJ, et al. Efficacy and safety of quetiapine in critically ill patients with delirium: a prospective, multicenter, randomized, double-blind, placebo-controlled pilot study. Crit Care Med. 2010;38(2):419-427. doi:10.1097/ CCM.0b013e3181b9e302

106. Dube KM, DeGrado J, Hohlfelder B, Szumita PM. Evaluation of the effects of quetiapine on QTc prolongation in critically ill patients. J Pharm Pract. 2018;31(3):292-297. doi:10.1177/ 0897190017711875

107. Fox MA, Elefritz JL, Huang BM, Hunley C. Comparison of lurasidone versus quetiapine for the treatment of delirium in critically ill patients. J Intensive Care Med. 2018;35:885066617754187.

108. Kim A, Lim KS, Lee H, et al. A thorough QT study to evaluate the QTc prolongation potential of two neuropsychiatric drugs, quetiapine and escitalopram, in healthy volunteers. Int Clin Psychopharmacol. 2016;31(4):210-217. doi:10.1097/YIC.0000000000000124

109. Lee S, Morris A, Kim S, Li F, Baumgartner L. Impact of quetiapine therapy on QTc prolongation in critically ill patients. Ann Pharmacother. 2019;53(7):705-710. doi:10.1177/1060028019829 494

110. Mangan KC, McKinzie BP, Deloney LP, Leon SM, Eriksson EA. Evaluating the risk profile of quetiapine in treating delirium in the intensive care adult population: a retrospective review. $J$ Crit Care. 2018;47:169-172. doi:10.1016/j.jcrc.2018.07.005

111. Nielsen J, Matz J, Mittoux A, et al. Cardiac effects of sertindole and quetiapine: analysis of ECGs from a randomized double-blind study in patients with schizophrenia. Eur Neuropsychopharmacol. 2015;25(3):303-311. doi:10.1016/j. euroneuro.2014.12.005
112. Potkin SG, Preskorn S, Hochfeld M, Meng X. A thorough QTc study of 3 doses of iloperidone including metabolic inhibition via CYP2D6 and/or CYP3A4 and a comparison to quetiapine and ziprasidone. $J$ Clin Psychopharmacol. 2013;33(1):3-10. doi:10.1097/JCP.0b013e31827c0314

113. Aghaienia N, Brahm NC, Lussier KM, Washington NB. Probable quetiapine-mediated prolongation of the QT interval. $J$ Pharm Pract. 2011;24(5):506-512. doi:10.1177/0897190011415683

114. Digby G, Machaalany J, Malik P, et al. Multifactorial QT interval prolongation. Cardiol J. 2010;17(2):184-188.

115. Furst BA, Champion KM, Pierre JM, Wirshing DA, Wirshing WC. Possible association of QTc interval prolongation with coadministration of quetiapine and lovastatin. Biol Psychiatry. 2002;51(3):264-265. doi:10.1016/S0006-3223(01)01333-6

116. Gupta A, Mody P, Pandey A. Inappropriate antibiotic therapy in a patient with heart failure and prolonged QT interval: a teachable moment. JAMA Intern Med. 2015;175(11):1748-1749. doi:10. 1001/jamainternmed.2015.5047

117. Hasnain M, Vieweg WV, Howland RH, et al. Quetiapine, QTc interval prolongation, and torsade de pointes: a review of case reports. Ther Adv Psychopharmacol. 2014;4(3):130-138. doi:10. 1177/2045125313510194

118. Vieweg WV, Schneider RK, Wood MA. Torsade de pointes in a patient with complex medical and psychiatric conditions receiving low-dose quetiapine. Acta Psychiatr Scand. 2005;112(4):318322; author reply 322. doi:10.1111/j.1600-0447.2005.00592.x

119. Vogel SM, Mican LM, Smith TL. Donepezil-induced QTc prolongation: a case report. Ment Health Clin. 2019;9(3):128-132. doi:10.9740/mhc.2019.05.128

120. Cartwright AL, Wilby KJ, Corrigan S, Ensom MH. Pharmacogenetics of risperidone: a systematic review of the clinical effects of CYP2D6 polymorphisms. Ann Pharmacother. 2013;47(3):350-360. doi:10.1345/aph.1R333

121. Gopal S, Hough D, Karcher K, et al. Risk of cardiovascular morbidity with risperidone or paliperidone treatment: analysis of 64 randomized, double-blind trials. J Clin Psychopharmacol. 2013;33(2):157-161. doi:10.1097/JCP.0b013e318283983f

122. Rabkin SW. Aging effects on QT interval: implications for cardiac safety of antipsychotic drugs. J Geriatr Cardiol. 2014;11 (1):20-25. doi:10.3969/j.issn.1671-5411.2014.01.005

123. Rattehalli RD, Jayaram MB, Smith M. Risperidone versus placebo for schizophrenia. Cochrane Database Syst Rev. 2010;(1): CD006918. doi:10.1002/14651858.CD006918

124. Azorin JM, Strub N, Loft H. A double-blind, controlled study of sertindole versus risperidone in the treatment of moderate-tosevere schizophrenia. Int Clin Psychopharmacol. 2006;21(1):4956. doi:10.1097/01.yic.0000177020.26311.a7

125. Llerena A, Berecz R, Dorado P, de la Rubia A. QTc interval, CYP2D6 and CYP2C9 genotypes and risperidone plasma concentrations. J Psychopharmacol. 2004;18(2):189-193. doi:10.11 $77 / 0269881104042618$

126. Ranjbar F, Akbarzadeh F, Ahmadi NM, Abbasnejhad M. Risperidone and corrected QT-interval prolongation in surface electrocardiogram. Pak J Biol Sci. 2012;15(10):496-500. doi:10.3923/pjbs.2012.496.500

127. Suzuki Y, Fukui N, Watanabe J, et al. QT prolongation of the antipsychotic risperidone is predominantly related to its 9hydroxy metabolite paliperidone. Hum Psychopharmacol. 2012;27(1):39-42. doi:10.1002/hup.1258

128. Suzuki Y, Tsuneyama N, Fukui N, et al. Effect of risperidone metabolism and P-glycoprotein gene polymorphism on QT interval in patients with schizophrenia. Pharmacogenomics $J$. 2014;14 (5):452-456. doi:10.1038/tpj.2014.6

129. Yerrabolu M, Prabhudesai S, Tawam M, Winter L, Kamalesh M. Effect of risperidone on QT interval and QT dispersion in the elderly. Heart Dis. 2000;2(1):10-12. 
130. Vieweg WV, Hasnain M, Hancox JC, et al. Risperidone, QTc interval prolongation, and torsade de pointes: a systematic review of case reports. Psychopharmacology (Berl). 2013;228(4):515524. doi:10.1007/s00213-013-3192-8

131. San-Juan-Rodriguez A, Zhang Y, He M, Hernandez I. Association of antidementia therapies with time to skilled nursing facility admission and cardiovascular events among elderly adults with alzheimer disease. JAMA Netw Open. 2019;2(3):e190213. doi:10.1001/jamanetworkopen.2019.0213

132. Gurbuz AS, Ozturk S, Acar E, et al. Acquired long QT syndrome and Torsades de Pointes related to donepezil use in a patient with Alzheimer disease. Egypt Heart J. 2016;68:197-199. doi:10. 1016/j.ehj.2015.07.004

133. Hadano Y, Ogawa H, Wakeyama T, et al. Donepezil-induced torsades de pointes without QT prolongation. J Cardiol Cases. 2013;8(2):e69-e71. doi:10.1016/j.jccase.2013.05.004

134. Kitt J, Irons R, Al-Obaidi M, Missouris C. A case of donepezilrelated torsades de pointes. BMJ Case Rep. 2015;2015. doi:10.1136/bcr-2015-211900

135. Leitch A, McGinness P, Wallbridge D. Calculate the QT interval in patients taking drugs for dementia. BMJ. 2007;335(7619):557. doi:10.1136/bmj.39020.710602.47

136. Takaya T, Okamoto M, Yodoi K, et al. Torsades de Pointes with QT prolongation related to donepezil use. J Cardiol. 2009;54 (3):507-511. doi:10.1016/j.jjcc.2009.03.011

137. Tanaka A, Koga S, Hiramatsu Y. Donepezil-induced adverse side effects of cardiac rhythm: 2 cases report of atrioventricular block and Torsade de Pointes. Intern Med. 2009;48(14):1219-1223. doi:10.2169/internalmedicine.48.2181

138. Sawada H, Oeda T, Kohsaka M, et al. Early use of donepezil against psychosis and cognitive decline in Parkinson's disease: a randomised controlled trial for 2 years. J Neurol Neurosurg Psychiatry. 2018;89(12):1332-1340. doi:10.1136/jnnp-2018-318 107

139. Fisher AA, Davis MW. Prolonged QT interval, syncope, and delirium with galantamine. Ann Pharmacother. 2008;42(2):278283. doi:10.1345/aph.1K514

140. Zhou T, Wang J, Xin C, Kong L, Wang C. Effect of memantine combined with citalopram on cognition of BPSD and moderate Alzheimer's disease: a clinical trial. Exp Ther Med. 2019;17 (3):1625-1630. doi:10.3892/etm.2018.7124
141. Seppi K, Ray chaudhuri K, Coelho M, et al. Update on treatments for nonmotor symptoms of Parkinson's disease-an evidence-based medicine review. Mov Disord. 2019;34(2):180-198. doi:10.1002/ mds. 27602

142. American Geriatrics Society. 2019 updated AGS beers criteria ${ }^{\circledR}$ for potentially inappropriate medication use in older adults. $J \mathrm{Am}$ Geriatr Soc. 2019;67(4):674-694. doi:10.1111/jgs.15767

143. Franchi C, Ardoino I, Rossio R, et al. Prevalence and risk factors associated with use of QT-prolonging drugs in hospitalized older people. Drugs Aging. 2016;33(1):53-61. doi:10.1007/s40266015-0337-y

144. Schachtele S, Tumena T, Gassmann KG, Fromm MF, Maas R. Co-prescription of QT-interval prolonging drugs: an analysis in a large cohort of geriatric patients. PLoS One. 2016;11(5): e0155649. doi:10.1371/journal.pone.0155649

145. McLean G, Hindle JV, Guthrie B, Mercer SW. Co-morbidity and polypharmacy in Parkinson's disease: insights from a large Scottish primary care database. BMC Neurol. 2017;17(1):126. doi:10.1186/s12883-017-0904-4

146. Santiago JA, Bottero V, Potashkin JA. Biological and clinical implications of comorbidities in Parkinson's disease. Front Aging Neurosci. 2017;9:394. doi:10.3389/fnagi.2017.00394

147. King LA, Priest KC, Nutt J, et al. Comorbidity and functional mobility in persons with Parkinson disease. Arch Phys Med Rehabil. 2014;95(11):2152-2157. doi:10.1016/j.apmr.2014.07. 396

148. Wang X, Zeng F, Jin WS, et al. Comorbidity burden of patients with Parkinson's disease and Parkinsonism between 2003 and 2012: a multicentre, nationwide, retrospective study in China. Sci Rep. 2017;7(1):1671. doi:10.1038/s41598-017-01795-0

149. Rautaharju PM, Surawicz B, Gettes LS, et al. AHA/ACCF/HRS recommendations for the standardization and interpretation of the electrocardiogram: part IV: the ST segment, T and U waves, and the QT interval: a scientific statement from the American Heart Association Electrocardiography and Arrhythmias Committee, Council on Clinical Cardiology; the American College of Cardiology Foundation; and the Heart Rhythm Society. Endorsed by the International Society for Computerized Electrocardiology. J Am Coll Cardiol. 2009;53(11):982-991. doi:10.1016/j.jacc.2008.12.014

\section{Publish your work in this journal}

Neuropsychiatric Disease and Treatment is an international, peerreviewed journal of clinical therapeutics and pharmacology focusing on concise rapid reporting of clinical or pre-clinical studies on a range of neuropsychiatric and neurological disorders. This journal is indexed on PubMed Central, the 'PsycINFO' database and CAS, and is the official journal of The International Neuropsychiatric Association (INA). The manuscript management system is completely online and includes a very quick and fair peer-review system, which is all easy to use. Visit http://www.dovepress.com/testimonials.php to read real quotes from published authors. 